\title{
Time-Dependent Long-Range-Corrected Double-Hybrid Density Functionals with Spin-Component and Spin-Opposite Scaling: A Comprehensive Analysis of Singlet-Singlet and Singlet-Triplet Excitation Energies
}

\author{
Marcos Casanova-Páez* and Lars Goerigk* \\ School of Chemistry, The University of Melbourne, Parkville, Victoria 3010, Australia \\ E-mail: mcasanova@student.unimelb.edu.au; lars.goerigk@unimelb.edu.au \\ Phone: +613834 46784
}

\begin{abstract}
Following the work on spin-component and spin-opposite scaled (SCS/SOS) global double hybrids for singlet-singlet excitations by Schwabe and Goerigk [J. Chem. Theory Comput. 2017, 13, 4307-4323] and our own works on new long-range corrected (LC) double hybrids for singlet-singlet and singlet-triplet excitations [J. Chem. Theory Comput. 2019, 15, 47354744; J. Chem. Phys. 2020, 153, 064106], we present new LC double hybrids with SCS/SOS that demonstrate further improvement over previously published results and methods. We introduce new unscaled and scaled versions of different global and LC double hybrids based on Becke88 or PBE exchange combined with LYP, PBE or P86 correlation. For singlet-singlet excitations, we cross-validate them on six benchmark sets that cover small to medium-sized chromophores with different excitation types (local valence, Rydberg, and charge transfer). For singlet-triplet excitations, we perform the cross-validation on three different benchmark sets following the same analysis as in our previous work in 2020. In total, 203 unique excitations are analyzed. Our results confirm and extend those of Schwabe and Goerigk regarding the superior performance of SCS and SOS variants compared to their unscaled parents by decreasing mean absolute deviations, root-mean-square deviations or error spans by more than half and bringing absolute mean deviations closer to zero. Our SCS/SOS variants show to be highly efficient and robust for the computation of vertical excitation energies, which even outperform specialized double hybrids that also contain an LC in their perturbative part. In particular, our new SCS/SOS- $\omega$ PBEPP86 and SCS/SOS- $\omega$ B88PP86 functional are four of the most accurate and robust methods tested in this work and we fully recommend them for future applications. However, if the relevant SCS and SOS algorithms are not available to the user, we suggest $\omega \mathrm{B} 88 \mathrm{PP} 86$ as the best unscaled method in this work.
\end{abstract}

\section{Introduction}

Density functional theory (DFT) is nowadays the most widely used methodology for the computation of ground state properties given its good compromise between accuracy and favorable scaling, making it possible to treat rela- tively large molecules, something that sometimes is infeasible for high-accurate, electron correlation wave function theory (WFT) methods. While its foundations, based on the Hohenberg-Kohn theorems ${ }^{1}$ and the KohnSham (KS) equations, $\frac{2}{2}$ are only valid for 
ground state properties, Runge and Gross formulated the equivalent of the HohenbergKohn theorems for the description of excited states in $1984, \frac{3}{}$ laying the foundations of time-dependent (TD) DFT. For weak external electromagnetic fields, linear-response TDDFT within the adiabatic approximation ${ }^{4}$ has become as important to many different fields working on electronically excited-state problems as DFT is the preferred method for ground state properties.

A ground state DFT calculation precedes the computation of electronic excitation energies within TD-DFT; therefore, both formalisms share the same shortcomings determined by the choice of the underlying density functional approximation (DFA) to the unknown "true" exchange-correlation functional. This work focuses on double-hybrid density functional approximations (DHDFAs) that follow Grimme's definition from 2006, $\stackrel{5}{ }$ whose outstanding performance have been demonstrated several times for ground state properties, as for example shown in Refs. 6-17. Shortly after the demonstrated success of the first DHDFA B2PLYP 5 for ground state properties, the DHDFA idea was extended to the computation of electronic vertical excitation energies in 2007 when Grimme and Neese ${ }^{18}$ combined Head-Gordon's configuration interaction singles with perturbative doubles [CIS(D) $]^{19}$ approach with the TD-DFT formalism and the closely related Tamm-Dancoff Approximation 20 (TDA). Herein, we exclusively deal with Grimme and Neese's approach, when we use the acronyms TD-DHDFAs and TDA-DHDFAs. We acknowledge other definitions of the DHDFA concept,, $21-25$ but also point out that not all of them are compatible with the TD(A)-DHDFA scheme proposed by Grimme and Neese, which is why they will not be considered in the present work.

Grimme and Neese's first TD(A)-B2PLYP study showed how the inclusion of the perturbative CIS(D) correction reduced the error span by about $0.3 \mathrm{eV}$ compared to the TD(A)DFT hybrid portion of B2PLYP.18 That work also showed promising results for singlet-triplet transitions in small (in)organic molecules and for doublet-doublet transitions in small radicals, which were limited to the TDA variant of B2PLYP. Subsequent applications on DHDFAs for excited states involved almost exclusively TD-DHDFA treatments of singlet-singlet transitions in organic systems. In 2009, the TDDHDFA idea was applied for the first time to B2GP-PLYP 26 on the famous test set containing small organic molecules proposed by Thiel and co-workers, ${ }^{27}$ where TD-B2GP-PLYP outperformed TD-B2PLYP and a range of lowerrung DFAs. 28 In 2013, the "non-empirical" functionals PBE0-DH ${ }^{29}$ and PBE0-2 $\sqrt[30]{ }$ were also assessed on the same test set in their TDA versions, but it was demonstrated that they were by on average up to $0.08 \mathrm{eV}$ less accurate than TD-B2(GP-)PLYP. ${ }^{31}$ Indeed, the label "non-empirical" should not be misunderstood as a synonym for quality or reliability, which was later demonstrated for DHDFAs applied to ground state properties, where semi-empirical DHDFAs by far outperformed non-empirical ones. 1032 Several studies have been conducted since Grimme and Neese's initial idea, and TD(A)-DHDFAs have been shown to be the most accurate DFAs for excited-state calculations and absorption spectra, 18/28/33,41] even rivaling standard WFT methods. 343941 While we refer the reader to a recently published detailed review on TD(A)-DHDFAs, 42 we highlight only some of the advantages of those over the conventional TD(A)-DFT approaches: a better description of electronic circular dichroism (ECD) spectra, 33 more accurate vertical singlet-singlet $18 / 28 / 34 / 39 \sqrt[43]{3}$ and singlettriplet 1814 excitations in organic moleculeswhere TD(A)-DHDFAs even outperformed the approximate coupled-cluster singles doubles WFT approach $\underline{4445}\left[\left(\mathrm{SCS}^{-}\right) \mathrm{CC} 2\right]^{34}$ a more balanced description of the problematic first two excited states in polycyclic aromatic hydrocarbons (PAHs), $35 \mid 40$ and no misleading results from artificial "ghost states".28|33|34|36 Another little-known but important finding in the context of ECD spectroscopy is that TD-DHDFAs were the only methods that could successfully reproduce an exciton-coupled spectrum of a merocyanine dimer aggregate,, 36 for which even long-range corrected (LC) (or range-separated) 
hybrid functionals failed and which initially had only been successfully described with WFT approaches. .46

A successful strategy to improve DHDFAs for ground state thermochemistry has been the introduction of spin-component and spinopposite scaling 47 49 (SCS/SOS) to the treatment of the non-local electron-correlation component. 6111 1412150151 SOS-based methods have the advantage of having a reduced formal scaling behavior if a Laplace transform algorithm is employed. ${ }^{48 \mid 52}$ We also mention in passing that the currently best-performing DHDFAs for molecular thermochemistry are the SCS-LC-DHDFAs $\omega$ DSD3-PBEPP86-D $4^{17}$ and $\omega \mathrm{DSD}_{72}$-PBEPP86-D4 ${ }^{16}$ based on studies with the GMTKN55 database for general maingroup thermochemistry, kinetics and noncovalent interactions; however, note that some of these methods follow different strategies compared to Grimme's original DHDFA definition from 2006.

In 2017, significant improvements were achieved by Schwabe and Goerigk when they successfully applied the SCS and SOS techniques to the CIS(D) component of global TD(A)-DHDFAs. A fitting procedure of the SCS/SOS parameters of six global DHDFAs was conducted against a high-level reference data training set. Successful cross-validation studies were carried out for vertical localvalence, vertical Rydberg, and 0-0 singletsinglet excitations using the TD- and TDADHDFA schemes. ${ }^{39}$ Scaled and unscaled DHDFAs were again superior to global and LC hybrids.

Their remarkable performance notwithstanding, global TD(A)-DHDFAs suffer from the same flaws as any other global DFA, $40141 / 43153 \sqrt[55]{5}$ namely the inability to adequately describe charge-transfer (CT) excitations. It was not until recently, in mid2019, that we closed this gap by introducing the first LC-DHDFAs optimized for vertical singlet-singlet excitation energies, namely TD- $\omega$ B2PLYP and TD- $\omega$ B2GP-PLYP, 40 both based on the original BLYP-based $[56 \mid 57$ global DHDFAs B2PLYP and B2GP-PLYP, respectively. Therein, we showed how the inclusion of a single parameter, which controls the interplay between the long- and short-range regimes, can improve long-range excitations such as Rydberg states and $\mathrm{CT}$ excitations without much loss in accuracy for local-valence transitions. We particularly note that both functionals delivered the best TD-DFT results to date for $\mathrm{L}_{\mathrm{a}}$ and $\mathrm{L}_{\mathrm{b}}$ transitions in PAHs, $\stackrel{40}{\underline{40}}$ and that they have already been successfully used in applications to systems of biological or technological relevance. 58,64

Prior to mid-2020, most of the BLYPbased global DHDFAs studies had been carried out using the full TD-DHDFA scheme, whereas most of the studies using PBE-based global DHDFAs had used the TDA-DHDFA scheme, instead. $37 / 38 / 65 / 75]$ Moreover, all previous benchmark studies and applications had been limited to singlet-singlet excitations, except for the original work by Grimme and Neese from $2007, \frac{18}{18}$ leaving out other spin multiplicities of crucial relevance, such as triplet excitations, most likely due to the lack of a code that could handle those transitions. For this reason, we assessed for the first time singlet-singlet and singlet-triplet excitations with global- and LC-DHDFAs based on both BLYP and PBE expressions for the underlying exchange-correlation functional and compared them with hybrid functionals. ${ }^{41}$ Our $\omega \mathrm{B} 2$ (GP-)PLYP and Adamo and co-workers' PBE-QIDH ${ }^{65}$ displayed the best results for singlet-triplet excitations, with the latter nevertheless still being unable to properly describe CT excitations, as we had to point out again in 2021. 43 A very important finding was that DHDFAs do not fix the triplet instability problem ${ }^{76} 79$ when the full TD-DHDFA scheme is applied, for which the TDA-DHDFA scheme must be employed instead.

Herein, we revisit the idea of applying SCS and SOS techniques to TD(A)-DHDFAs similar to the work done by Schwabe and Goerigk, and we extend them even further by applying the latest TD(A)-LC-DHDFAs not only for singlet-singlet excitations but also to singlettriplet excitations. For this purpose, we analyze 22 different scaled global- and LC-DHDFAs. Among the 8 unscaled DHDFAs, three are 
global and five are LC ones. 6 of those unscaled methods had been analyzed by us before, but we also present two new LC-DHDFAs named $\omega \mathrm{PBEPP} 86$ and $\omega \mathrm{B} 88 \mathrm{PP} 86$. The remaining 14 methods are newly developed scaled versions of the 8 unscaled ones. Lastly, we also compare those 22 methods with Mester and Kállay's three newest "Range-Separated Double-Hybrid functional" (RS-DH) methods. ${ }^{80}$ The novelty of these RS-DHs is that they have been designed to be long-range corrected not only in the exchange component (as usual LC-methods) but also in the perturbative non-local CIS(D) correction. Note that in their original work, they only analyzed singlet-singlet excitations using the TDA-DHDFA scheme and our comparison will be limited to those values.

In this work, we briefly review the theoretical background required for our new method developments. We then discuss the definition of our new unscaled LC-DHDFAs $\omega$ B88PP86 and $\omega$ PBEPP86, followed by the development of SCS and SOS extensions for each tested method in this work (except the RS-DH methods). The new SCS and SOS TD(A)-DHDFAs are then cross-validated in a series of benchmark sets that cover singlet-singlet and singlet-triplet excitations. We finally conclude with a discussion of the general robustness of the newly proposed methods and some final remarks for the user community.

\section{Theoretical Background}

\subsection{Linear-Response TD-DFT}

The most common method for computing excited-state properties is linear-response 81 time-dependent TD-DFT within the adiabatic approximation. $\frac{484}{84}$ The latter allows us to replace the time-dependent exchangecorrelation kernel with the time-independent one from ground state DFT. The technicalities of TD-DFT are well-documented in the literature, 18394073 which is why we keep our explanations as brief as possible. For real orbitals, the vertical excitation energies are computed as the solution of the random-phase- approximation (RPA)-type eigenvalue problem: 85

$$
\left(\begin{array}{cc}
\mathbf{A} & \mathbf{B} \\
\mathbf{B} & \mathbf{A}
\end{array}\right)\left(\begin{array}{c}
\mathbf{X}^{(\mathrm{n})} \\
\mathbf{Y}^{(\mathrm{n})}
\end{array}\right)=\Delta \mathrm{E}^{(\mathrm{n})}\left(\begin{array}{cc}
\mathbf{1} & \mathbf{0} \\
\mathbf{0} & -\mathbf{1}
\end{array}\right)\left(\begin{array}{c}
\mathbf{X}^{(\mathrm{n})} \\
\mathbf{Y}^{(\mathrm{n})}
\end{array}\right),
$$

$\Delta \mathrm{E}^{(\mathrm{n})}$ is the vertical excitation energy for the $\mathrm{n}^{\text {th }}$ excited state, with $\mathbf{X}^{(\mathrm{n})}$ and $\mathbf{Y}^{(\mathrm{n})}$ being the corresponding eigenvectors for single-particle excitations and de-excitations, respectively. A and $\mathbf{B}$ are matrices that contain the information on these excitations and de-excitations, whose components are given by: 83

$$
\begin{aligned}
& \mathrm{A}_{\mathrm{ia} \sigma, \mathrm{jb} \tau}=\delta_{\sigma \tau} \delta_{\mathrm{ij}} \delta_{\mathrm{ab}}\left(\varepsilon_{\mathrm{a} \sigma}-\varepsilon_{\mathrm{i} \tau}\right)+\left(\mathrm{i}_{\sigma} \mathrm{a}_{\sigma} \mid \mathrm{j}_{\tau} \mathrm{b}_{\tau}\right) \\
& -\delta_{\sigma \tau} \mathrm{a}_{\mathrm{x}}\left(\mathrm{i}_{\sigma} \mathrm{j}_{\sigma} \mid \mathrm{a}_{\tau} \mathrm{b}_{\tau}\right)+\left(1-\mathrm{a}_{\mathrm{x}}\right)\left(\mathrm{i}_{\sigma} \mathrm{a}_{\sigma}\left|\mathrm{f}^{\mathrm{XC}}\right| \mathrm{j}_{\tau} \mathrm{b}_{\tau}\right)
\end{aligned}
$$

and

$$
\begin{aligned}
B_{i a \sigma, j b \tau}= & \left(i_{\sigma} a_{\sigma} \mid b_{\tau} j_{\tau}\right)-\delta_{\sigma \tau} a_{x}\left(i_{\sigma} b_{\sigma} \mid a_{\tau} j_{\tau}\right) \\
& +\left(1-a_{x}\right)\left(i_{\sigma} a_{\sigma}\left|f^{X C}\right| b_{\tau} j_{\tau}\right) .
\end{aligned}
$$

As usual, $\sigma$ and $\tau$ are spin variables, the indices $\mathrm{i}, \mathrm{j}, \mathrm{k} \ldots$ in this paper refer to occupied orbitals, a, b, c...to virtual orbitals, and ax is the Fock exchange scaling parameter. The last term in these equations has DFT origin, where $\mathrm{f}^{\mathrm{XC}}$ is the exchange-correlation kernel and defined as the second derivative of the exchangecorrelation functional $\mathrm{E}_{\mathrm{XC}}$ with respect to the electronic density $\rho$ :

$$
\mathrm{f}^{\mathrm{XC}}\left(\mathbf{r}_{1}, \mathbf{r}_{2}\right)=\frac{\partial^{2} \mathrm{E}_{\mathrm{XC}}}{\partial \rho\left(\mathbf{r}_{1}\right) \partial \rho\left(\mathbf{r}_{2}\right)}
$$

with $\mathbf{r}_{1 / 2}$ being spatial variables.

By neglecting the $\mathbf{B}$ matrix from Eq.(1), we can obtain a simplified version known as the Tamm-Dancoff approximation (TDA), 20 resulting in an eigenvalue problem that reads

$$
\mathbf{A} \mathbf{X}^{(\mathrm{n})}=\Delta \mathrm{E}^{(\mathrm{n})} \mathbf{X}^{(\mathrm{n})}
$$

The label (n) will be dropped in the following.

\subsection{Vertical Excitation Energies with DHDFAs}

We follow Grimme's original definition of DHDFAs ${ }^{18}$ alongside the LC scheme, ${ }^{86}$ similar as in 
our construction of $\omega \mathrm{B} 2(\mathrm{GP}-) \mathrm{PLYP}: \underline{40}$

$$
\begin{aligned}
\mathrm{E}_{\mathrm{XC}}^{\mathrm{LC}-\mathrm{DHDF}} & =\mathrm{ax}_{\mathrm{X}}^{\mathrm{SR}-\mathrm{HF}}(\omega)+\mathrm{ax}_{\mathrm{X}, \mathrm{DFA}} \mathrm{E}_{\mathrm{X}}^{\mathrm{SR}-\mathrm{DFT}}(\omega) \\
& +\mathrm{E}_{\mathrm{X}}^{\mathrm{LR}-\mathrm{HF}}(\omega)+\mathrm{a}_{\mathrm{C}, \mathrm{DFA}} \mathrm{E}_{\mathrm{C}}^{\mathrm{DFT}}+\mathrm{a}_{\mathrm{C}} \mathrm{E}_{\mathrm{C}}^{\mathrm{PT} 2},
\end{aligned}
$$

where the first term corresponds to shortrange Fock exchange, followed by the shortrange DFT-exchange functional. The third term is the full, long-range Fock exchange to ensure the correct $1 / \mathrm{r}_{12}$ decay in the potential, where $r_{12}$ is the interelectronic distance. The last two terms are the DFT-correlation functional and the second-order perturbative, nonlocal-correlation correction given by $\mathrm{MP} 2 \underline{87}$ for ground state or CIS(D) for excitation energy calculations. Finally, ax,DFA and $\mathrm{a}_{\mathrm{C}, \mathrm{DFA}}$ are scaling parameters for DFT exchange and correlation, whereas $\mathrm{ax}_{\mathrm{X}}$ and $\mathrm{a}_{\mathrm{C}}$ scale shortrange Fock exchange and non-local correlation, respectively; usually, but not always, those parameter values depend on one another. 5293165188 The interplay between shortand long-range regimes is controlled by the range-separation parameter $\omega$.

In the spirit of ground state DHDFA calculations, Grimme and Neese's vertical excitation energies at the double-hybrid level $\left(\Delta \mathrm{E}_{\mathrm{TD}}(\mathrm{A})-\mathrm{DHDFA}\right)$ are obtained in a two-step procedure, $\frac{18}{18}$ first, we solve either the full TD(Eq.1) or TDA-DFT (Eq.5) eigenvalue problem using only the hybrid part of the DHDFA, i.e., all terms in Eq. 6] excluding the perturbative portion. This gives rise to a vertical excitation energy of hybrid-DFT quality $\left(\Delta \mathrm{E}_{\mathrm{TD}(\mathrm{A}) \text {-hybrid }}\right)$, which is then perturbatively corrected with Head-Gordon's CIS(D) correction $\left(\Delta_{\mathrm{CIS}(\mathrm{D})}\right): \underline{19}$

$$
\Delta \mathrm{E}_{\mathrm{TD}(\mathrm{A})-\mathrm{DHDFA}}=\Delta \mathrm{E}_{\mathrm{TD}(\mathrm{A}) \text {-hybrid }}+\mathrm{a}_{\mathrm{C}} \Delta_{\mathrm{CIS}(\mathrm{D})},
$$

where the parameter $\mathrm{a}_{\mathrm{C}}$ scales the CIS(D)type contribution. More details on TD(A)DHDFAs are given in our recent free-access account in Ref. 42 .

\subsection{The CIS(D) Correction}

The CIS(D) formalism for TD(A)-DHDFAs has been extensively explained in the literature and we refer the reader to Refs 18 and 39 for more detailed information. In the spirit of MP2 being a second-order perturbative correlation correction to a Hartree-Fock (HF) ground state energy, the second-order perturbative CIS(D) correction 19 introduces some electron correlation to configuration interaction singles (CIS) excitation energies. The CIS(D) energy correction to the total CIS energy of an excited state is:

$$
\mathrm{E}_{\mathrm{C}}^{\mathrm{CIS}(\mathrm{D})}=\left\langle\Phi_{\mathrm{CIS}}|\hat{\mathrm{V}}| \hat{\mathrm{U}}_{2} \Phi_{0}\right\rangle+\left\langle\Phi_{\mathrm{CIS}}|\hat{\mathrm{V}}| \hat{\mathrm{T}}_{2} \hat{\mathrm{U}}_{1} \Phi_{0}\right\rangle
$$

where the first and second terms are known as the "direct" and "indirect" terms, respectively. $\hat{U}_{1}$ and $\hat{U}_{2}$ are operators that generate the singly- and doubly-excited wave functions from the HF determinant $\left(\Phi_{0}\right), \hat{\mathrm{T}}_{2}$ is the operator that generates the double excitation of two CIS-inactive electrons, and $\hat{\mathrm{V}}$ is a perturbation potential. $\Phi_{\text {CIS }}$ is the CIS wave function according to:

$$
\Phi_{\mathrm{CIS}}=\hat{\mathrm{U}}_{1} \Phi_{0}=\sum_{\mathrm{ia}} \mathrm{t}_{\mathrm{i}}^{\mathrm{a}} \Phi_{\mathrm{i}}^{\mathrm{a}},
$$

where $\mathrm{t}_{\mathrm{i}}^{\mathrm{a}}$ are the CIS single-excitation amplitudes and $\Phi_{\mathrm{i}}^{\mathrm{a}}$ is the singly excited determinant. The $\hat{\mathrm{T}}_{2}$ operator acting on the HF determinant, $\Phi_{0}$, is given by

$$
\begin{aligned}
\hat{\mathrm{T}}_{2} \Phi_{0} & =\frac{1}{4} \sum_{\mathrm{ijab}} \mathrm{t}_{\mathrm{ij}}^{\mathrm{ab}} \Phi_{\mathrm{ij}}^{\mathrm{ab}} \\
& =-\frac{1}{4} \sum_{\mathrm{ijab}} \frac{(\mathrm{ij} \| \mathrm{ab})}{\varepsilon_{\mathrm{a}}+\varepsilon_{\mathrm{b}}-\varepsilon_{\mathrm{i}}-\varepsilon_{\mathrm{j}}} \Phi_{\mathrm{ij}}^{\mathrm{ab}},
\end{aligned}
$$

where we have used Mulliken's notation for the antisymmetrized two-electron integral $(\mathrm{ij} \| \mathrm{ab})=(\mathrm{ij} \mid \mathrm{ab})-(\mathrm{ia} \mid \mathrm{jb})$ and $\varepsilon$ stands again for the orbital energy. $\Phi_{\mathrm{ij}}^{\mathrm{ab}}$ is the doubly-excited determinant from the $\mathrm{HF}$ ground state created by the operator $\hat{U}_{2}$ :

$$
\hat{\mathrm{U}}_{2} \Phi_{0}=-\frac{1}{4} \sum_{\mathrm{ijab}} \frac{\left\langle\Phi_{\mathrm{ij}}^{\mathrm{ab}}|\hat{\mathrm{V}}| \hat{\mathrm{U}}_{1} \Phi_{0}\right\rangle}{\varepsilon_{\mathrm{a}}+\varepsilon_{\mathrm{b}}-\varepsilon_{\mathrm{i}}-\varepsilon_{\mathrm{j}}-\Delta \mathrm{E}_{\mathrm{CIS}}} \Phi_{\mathrm{ij}}^{\mathrm{ab}}
$$

The actual CIS(D) correction, $\Delta_{\mathrm{CIS}(\mathrm{D})}$, to the CIS excitation energy, $\Delta \mathrm{E}_{\mathrm{CIS}}$, is obtained as the difference between the second-order perturbative correlation energies for the excited 
and the ground states:

$$
\begin{aligned}
\Delta_{\mathrm{CIS}(\mathrm{D})} & =\mathrm{E}_{\mathrm{C}}^{\mathrm{CIS}(\mathrm{D})}-\mathrm{E}_{\mathrm{C}}^{\mathrm{MP} 2} \\
& =-\frac{1}{4} \sum_{\mathrm{ijab}} \frac{\left(\mathrm{u}_{\mathrm{ij}}^{\mathrm{ab}}\right)^{2}}{\varepsilon_{\mathrm{a}}+\varepsilon_{\mathrm{b}}-\varepsilon_{\mathrm{i}}-\varepsilon_{\mathrm{j}}-\Delta \mathrm{E}_{\mathrm{CIS}}}+\sum_{\mathrm{i} \mathrm{a}} \mathrm{t}_{\mathrm{i}}^{\mathrm{a}} \mathrm{v}_{\mathrm{i}}^{\mathrm{a}},
\end{aligned}
$$

with the corresponding definitions

$$
\mathrm{u}_{\mathrm{ij}}^{\mathrm{ab}}=\sum_{\mathrm{c}}(\mathrm{ab} \| \mathrm{cj}) \mathrm{t}_{\mathrm{i}}^{\mathrm{c}}-(\mathrm{ab} \| \mathrm{ci}) \mathrm{t}_{\mathrm{j}}^{\mathrm{c}}-\sum_{\mathrm{k}}(\mathrm{ka} \| \mathrm{ij}) \mathrm{t}_{\mathrm{k}}^{\mathrm{b}}-(\mathrm{kb} \| \mathrm{ij}) \mathrm{t}_{\mathrm{k}}^{\mathrm{a}}
$$

and

$$
\mathrm{v}_{\mathrm{i}}^{\mathrm{a}}=\frac{1}{2} \sum_{\mathrm{jkbc}}(\mathrm{jk} \| \mathrm{bc})\left[\mathrm{t}_{\mathrm{i}}^{\mathrm{b}} \mathrm{t}_{\mathrm{jk}}^{\mathrm{ca}}+\mathrm{t}_{\mathrm{j}}^{\mathrm{a}} \mathrm{t}_{\mathrm{ik}}^{\mathrm{cb}}+2 \mathrm{t}_{\mathrm{j}}^{\mathrm{b}} \mathrm{t}_{\mathrm{ik}}^{\mathrm{ac}}\right]
$$

A TD(A)-DHDFA excitation energy is calculated by employing Eq.12 with KS orbitals instead of the HF ones and with $\Delta \mathrm{E}_{\mathrm{TD}}(\mathrm{A})$-hybrid excitation energies instead of $\Delta \mathrm{E}_{\mathrm{CIS}}$. For TDADHDFA, it is straightforward to employ the $\mathbf{X}$ vector (Eq.5) for the single-excitation amplitudes $\mathrm{t}_{\mathrm{i}}^{\mathrm{a}}$, whereas for the full TD-DHDFA the $\mathbf{X}$ vector is employed but the $\mathbf{Y}$ vector neglected (Eq.1).

\subsection{The SCS- and SOS-CIS(D) Variants}

Similar to Grimme's SCS-MP2 method, 47 the electron pair contributions to the correlation energy of an excitation energy can be separated into same (SS) and opposite spin (OS) contributions, which are then scaled differently . The first implementation of the SCS-CIS(D) idea dates back to 2004 when Grimme and Izgorodina applied the same scaling parameters as in SCS-MP2 ground state theory to the "indirect" term in Eq. 8, ${ }^{89}$ Later, Rhee and Head-Gordon extended this idea and applied additional scale parameters to the "direct" term. .90 Therefore, we can break down the two terms in Eq. (8) into same- and opposite spin components according to:

$$
\begin{aligned}
& \mathrm{E}_{\mathrm{c}}^{\mathrm{SCS}-\mathrm{CIS}(\mathrm{D})}=\left\langle\Phi_{\mathrm{CIS}}|\hat{\mathrm{V}}|\left(\mathrm{c}_{\mathrm{U}}^{\mathrm{OS}} \hat{\mathrm{U}}_{2}^{\mathrm{OS}}+\mathrm{c}_{\mathrm{U}}^{\mathrm{SS}} \hat{\mathrm{U}}_{2}^{\mathrm{SS}}\right) \Phi_{0}\right\rangle \\
& +\left\langle\Phi_{\mathrm{CIS}}|\hat{\mathrm{V}}|\left(\mathrm{c}_{\mathrm{T}}^{\mathrm{OS}} \hat{\mathrm{T}}_{2}^{\mathrm{OS}}+\mathrm{c}_{\mathrm{T}}^{\mathrm{SS}} \hat{\mathrm{T}}_{2}^{\mathrm{SS}}\right) \hat{\mathrm{U}}_{1} \Phi_{0}\right\rangle,
\end{aligned}
$$

where $\mathrm{c}_{\mathrm{U}}^{\mathrm{SS}}$ and $\mathrm{c}_{\mathrm{U}}^{\mathrm{OS}}$ are the same- and oppositespin scale parameters for the direct term and
$\mathrm{c}_{\mathrm{T}}^{\mathrm{SS}}$ and $\mathrm{c}_{\mathrm{T}}^{\mathrm{OS}}$ the related parameters for the indirect term. We refer the reader to e.g. Ref. 39 for the explicit form of the SS and OS terms shown in Eq. 15). By setting the SS parameters to zero, one reduces the SCS- to the SOSCIS(D) approach, which can benefit from a better formal scaling behavior in the same way as SOS-MP2. 48

Coming back to TD(A)-DHDFs, the expression for the SCS/SOS-CIS(D) correction $\left(\Delta_{\mathrm{SCS} / \mathrm{SOS}-\mathrm{CIS}(\mathrm{D})}\right)$, which is added to the excitation energy of the hybrid portion of the DHDFA, follows the same structure as $\Delta_{\mathrm{CIS}(\mathrm{D})}$ in Eq. (7), once the four SS/OS terms are taken into consideration, giving the $\mathrm{TD}(\mathrm{A})$ SCS/SOS-DHDF excitation energy:

$\Delta \mathrm{E}_{\mathrm{TD}(\mathrm{A})-\mathrm{SCS} / \mathrm{SOS}-\mathrm{DHDFA}}=\Delta \mathrm{E}_{\mathrm{TD}(\mathrm{A}) \text {-hybrid }}+\Delta_{\mathrm{SCS} / \mathrm{SOS}-\mathrm{CIS}(\mathrm{D})}$.

Note that in the work by Rhee and Head-

Gordon, the definition of the $\mathrm{U}_{2}^{\mathrm{SS} / \mathrm{OS}}$ operator considers an extra "damping" parameter $(\lambda)$ for $\Delta \mathrm{E}_{\mathrm{CIS}}$ and two different versions of SCSCIS(D) were developed by setting $\lambda=0$ and $\lambda=1$. Herein, we set this parameter to unity following the same protocol as in Refs. 34 and 39.

\section{Computational Details}

All molecular geometries were taken from the literature, and all calculations were performed with the same AO basis set used in the respective works that developed the reference values to enable a direct comparison. In this context, we refrain from any basis set dependence study as it has already been conducted. 39 It has been established that even for full CIS(D) $\left(\mathrm{a}_{\mathrm{C}}=1\right)$, local-valence and Rydberg excitation energies only differed by about $0.03 \mathrm{eV}$ when going from a triple to a large quadruple- $\zeta$ basis set. Unsurprisingly, this difference was even lower for DHDFAs with smaller $\mathrm{a}_{\mathrm{C}}$. For instance, there was an average difference of just $0.01 \mathrm{eV}$ for TD-B2PLYP $\left(\mathrm{a}_{\mathrm{C}}=0.27\right)$ and TDPBE0-DH $\left(\mathrm{a}_{\mathrm{C}}=0.125\right) . .39$ We implemented the SCS/SOS-CIS(D) code for singlet and triplet excitations into a local version of the ORCA code,, 93 and it will be available in the upcoming release of ORCA5. The SCF convergence 
criterion was set to $10^{-7} \mathrm{E}_{\mathrm{h}}\left(10^{-8} \mathrm{E}_{\mathrm{h}}\right.$ for the updated Gordon set) along with ORCA's numerical quadrature grid "4" (grid "5" and "finalgrid 6" for the updated Gordon set). The resolutionof-the-identity technique was used with appropriate auxiliary basis sets for the perturbative steps. 9495

Hybrid density functionals are not assessed in this work because we aim to improve our previously published DHDFA results by introducing two new exchange-correlation combinations and also the SCS/SOS technique. We refer the reader to our previous works in Refs. 40 and 41 for more information about the performance of global and LC hybrid functionals, which are shown to be less accurate and robust; in this context, we would just like to mention that the LC hybrid $\omega$ B97 ${ }^{96}$ sometimes outperformed some DHDFAs such as B2PLYP, PBE0-DH, 29 and RSX-QIDH, 97 but not other DHDFAs tested by us. Due to a lack of highlevel reference data for triplet excitations in the PAH and CT sets, we expand our analysis by also assessing the full TD-DHDFA scheme on these two sets without any further optimization procedure. In addition, we also assessed the new RS-DH methods by Mester and Kállay, $\underline{80}$ namely, RS-B88-LYP, RS-PBE-PBE, and RS-PBE-P86 which had been optimized with Schwabe and Goerigk's "updated Gordon set" 3998 for singlet-singlet transitions using the TDA-DHDFA scheme; note that for these methods, we took the relevant values directly from the original work.

It is also important to consider the definition of "chemical accuracy" for excitation energy calculations. It is a somewhat arbitrarily defined value and some studies have suggested a value of $0.05 \mathrm{eV}, \underline{6799}$ whereas others prefer to regard an accuracy of $0.1 \mathrm{eV}$ as a success. 28100 Our study can be interpreted within the context of either of these two definitions.

Table 1: Optimized parameters for our new LCDHDFAs following Eq. .

\begin{tabular}{cccccc}
\hline Functional & $\mathrm{a}_{\mathrm{X}}$ & $\mathrm{ax}_{\mathrm{X}, \mathrm{DFA}}$ & $\mathrm{a}_{\mathrm{C}, \mathrm{DFA}}$ & $\mathrm{a}_{\mathrm{C}}$ & $\omega\left[\mathrm{bohrs}^{-1}\right]$ \\
\hline$\omega \mathrm{B} 88 \mathrm{PP} 86$ & 0.65 & 0.35 & 0.58 & 0.42 & 0.20 \\
$\omega$ PBEPP86 & 0.70 & 0.30 & 0.68 & 0.48 & 0.18 \\
\hline
\end{tabular}

\section{Development}

\subsection{Introducing $\omega \mathrm{B} 88 \mathrm{PP} 86$ and wPBEPP86}

Our new $\omega$ B88PP86 and $\omega$ PBEPP86 functionals are based on Becke88 and PBE exchange, respectively, however with modifications proposed by Hirao and co-workers $\sqrt{86}$ in order to make them applicable within the LC scheme. Both methods rely on Perdew's correlation functional from 1986 (P86) 101 with the Vosko, Wilk, and Nusair "VWN-V" local spin-density component 102 The choice of those exchange and correlation functionals is based on the good performance of the global PBE-based DHDFA from our previous study, 11 namely PBE-QIDH, where it was able to properly describe Rydberg transitions, the RS-PBE-P86 method that performed well for the updated Gordon test set, $\underline{80}$ and the DSD-PBEP $86^{12}$ ground state DHDFA that performed well for ground state thermochemistry ${ }^{8}$ and some excited-state properties. 39

Both methods follow the LC-DHDFA definition from Eq.6 and were optimized for singletsinglet excitations, using the TDA-DHDFA scheme, by minimizing the root-mean-square deviation (RMSD) for the well-balanced updated Gordon set. We performed the optimization for singlet-singlet excitations using only the TDA-DHDFA scheme due to its more affordable computational cost and to make them applicable to singlet-triplet excitations, based on our previous experience with TDA- and TD-DHDFAs. ${ }^{41}$ We optimized these methods only for singlet-singlet transitions to make them comparable to previously published methods. The original benchmark set by Gordon and co-workers ${ }^{98}$ comprised a total of 63 vertical singlet-singlet excitations made up of 32 valence and 31 Rydberg states in 14 different molecules but was based on different types of geometries and experimental reference values. Schwabe and Goerigk re-optimized the geometries with a consistent level of theory in 2017, $\underline{39}$ and also provided new full and estimated Approximate Coupled-Cluster Singles Doubles Triples data (CC3 $3^{103} /$ aug-cc-pVTZ ${ }^{104 / 105}$ ). 
For the optimization procedure of $\omega \mathrm{B} 88 \mathrm{PP} 86$ and $\omega$ PBEPP86, we set the initial scaling parameters for the DFT, Fock exchange, and perturbative correlation components to those of B2GP-PLYP and PBE-QIDH, respectively. Those starting parameters were chosen, as both functionals performed reasonably well for localvalence excitations. We then optimized $\omega$. Finally, a "full" optimization of all remaining scale parameters was carried out for that $\omega$ value, giving rise to the final values presented in Table 1.

Interestingly, our resulting scale parameters for Fock exchange and perturbative correlation follow Toulouse et al.'s one-parameter quadratic relation $\left(\mathrm{a}_{\mathrm{X}}^{2}=\mathrm{a}_{\mathrm{C}}\right), \frac{88}{88}$ even though the latter had not been derived for excitation energies. Results for the new methods for the fit set will be discussed further below after the introduction of the new SCS/SOS methods.

\subsection{New TDA-DHDFAs with SCS and SOS Components for Ex- citation Energies}

\subsubsection{Fitting Procedure}

We combined the TD(A)-DHDFA idea with the SCS/SOS-CIS(D) approach as presented earlier in Eq. 16. For that purpose, we considered eight different unscaled DHDFA methods: B2PLYP, ${ }^{5}$ B2GP-PLYP, 26 $\omega B 2 P L Y P, 40$ $\omega$ B2GP-PLYP, 40 PBE-QIDH, 65 RSX-QIDH, ${ }^{97} \omega \mathrm{B} 88 \mathrm{PP} 86$, and $\omega \mathrm{PBEPP} 86$. According to our previous work in Ref. 41, PBE0-DH and RSX-0DH ${ }^{106}$ do not perform well, particularly the latter, which is why we refrained from including them in this work. Since SCS and SOS variants of B2PLYP and B2GPPLYP have already been presented in Ref. 39, we will label our new variants as SCS/SOSB2PLYP21 and SCS/SOS-B2GP-PLYP21, respectively. We re-optimized these two DHDFAs given that this time we also include singlettriplet excitations in the fitting procedure (see below). For each one of these functionals, we analyzed two different flavors for the scaling parameters:
- A free fit of all four parameters (SCS variant).

- An SOS variant with $\mathrm{c}_{\mathrm{T}}^{\mathrm{SS}}=\mathrm{c}_{\mathrm{U}}^{\mathrm{SS}}=0$ and a free fit of the remaining two OS parameters.

On rare occasions, the free fit of the four parameters gave negative, unphysical values for one or both SS parameters. In those cases, we set those parameters to zero. In two cases, namely B2PLYP and $\omega$ B2PLYP, the SCS fit led to the SOS variant. Hence, the subsequently used label "SCS/SOS-B2PLYP21" refers to one and the same method; the same is true for "SCS/SOS- $\omega$ B2PLYP.

The fitting procedure was conducted with the full updated Gordon benchmark set. This includes the 63 vertical singlet-singlet excitations mentioned in Section 4.1 and additional data for singlet-triplet excitations developed as part of Ref. 39 and published for the first time by us in mid-2020. 41 Those 38 vertical singlettriplet excitations are made up of 27 valence and 11 Rydberg excitations from 12 different molecules, all with the same full or estimated high-level CC3/aug-cc-pVTZ reference data.

Our parametrization procedure consisted of a minimization of the RMSD for each method on the entire set. The final optimized scaling parameters are shown in Table2, Therein, we also provide a breakdown into which DHDFAs are global and which are long-range corrected. We analyze the singlet-singlet and singlet-triplet excitation energies for the fit set separately in the following two sections.

\subsubsection{Analysis of Vertical Singlet- Singlet Excitations}

Our results for the singlet excitations in the fit sets are broken down into the whole set and its local-valence and Rydberg components. All relevant statistical results are shown in Table 3 for each TDA-DHDFA method. First of all, we would like to point out that many methods share the same RMSDs. However, to be able to rank them, we decided to sort the methods according to the following criteria: RMSD, MAD, 
Table 2: Original $^{\mathrm{a}}$ parameters of unscaled DHDFAs and parameters of their new SCS and SOS variants optimized for the TDA-DHDFA scheme.

\begin{tabular}{|c|c|c|c|c|c|}
\hline Method & & $\mathrm{c}_{\mathrm{T}}^{\mathrm{SS}}$ & $\mathrm{c}_{\mathrm{T}}^{\mathrm{OS}}$ & $\mathrm{c}_{\mathrm{U}}^{\mathrm{SS}}$ & $\mathrm{c}_{\mathrm{U}}^{\mathrm{OS}}$ \\
\hline \multirow{3}{*}{$\mathrm{B} 2 \mathrm{PLYP} \mathrm{P}^{\mathrm{b}}$} & Original & 0.270 & 0.270 & 0.270 & 0.270 \\
\hline & $\mathrm{SCS}^{\mathrm{c}}$ & 0.000 & 0.334 & 0.000 & 0.283 \\
\hline & $\operatorname{sOS}^{c}$ & 0.000 & 0.334 & 0.000 & 0.283 \\
\hline \multirow{3}{*}{ B2GP-PLYP ${ }^{b}$} & Original & 0.360 & 0.360 & 0.360 & 0.360 \\
\hline & SCS & 0.018 & 0.475 & 0.000 & 0.468 \\
\hline & SOS & 0.000 & 0.489 & 0.000 & 0.484 \\
\hline \multirow{3}{*}{ PBE-QIDH $^{\mathrm{b}}$} & Original & 0.330 & 0.330 & 0.330 & 0.330 \\
\hline & SCS & 0.070 & 0.515 & 0.096 & 0.524 \\
\hline & SOS & 0.000 & 0.547 & 0.000 & 0.573 \\
\hline \multirow{3}{*}{$\omega \mathrm{B} 2 \mathrm{PLYP} \mathrm{P}^{\mathrm{d}}$} & Original & 0.270 & 0.270 & 0.270 & 0.270 \\
\hline & $\mathrm{SCS}^{\mathrm{C}}$ & 0.000 & 0.433 & 0.000 & 0.460 \\
\hline & $\mathrm{SOS}^{\mathrm{c}}$ & 0.000 & 0.433 & 0.000 & 0.460 \\
\hline \multirow{3}{*}{$\omega B 2 G P-P L Y P^{d}$} & Original & 0.360 & 0.360 & 0.360 & 0.360 \\
\hline & SCS & 0.000 & 0.571 & 0.135 & 0.558 \\
\hline & SOS & 0.000 & 0.570 & 0.000 & 0.610 \\
\hline \multirow{3}{*}{ RSX-QIDH ${ }^{\mathrm{d}}$} & Original & 0.330 & 0.330 & 0.330 & 0.330 \\
\hline & SCS & 0.174 & 0.541 & 0.586 & 0.424 \\
\hline & SOS & 0.000 & 0.632 & 0.000 & 0.714 \\
\hline \multirow{3}{*}{$\omega \mathrm{B} 88 \mathrm{PP} 86^{\mathrm{d}}$} & Original & 0.423 & 0.423 & 0.423 & 0.423 \\
\hline & SCS & 0.000 & 0.557 & 0.092 & 0.545 \\
\hline & SOS & 0.000 & 0.559 & 0.000 & 0.599 \\
\hline \multirow{3}{*}{$\omega$ PBEPP $86^{\mathrm{d}}$} & Original & 0.480 & 0.480 & 0.480 & 0.480 \\
\hline & SCS & 0.000 & 0.610 & 0.297 & 0.540 \\
\hline & SOS & 0.000 & 0.613 & 0.000 & 0.669 \\
\hline
\end{tabular}

${ }^{a}$ If all four scaling parameters are identical, the original, unscaled DHDF is obtained, as all parameters equal $\mathrm{a}_{\mathrm{C}}$ in Eq. 7. ${ }^{\mathrm{b}}$ Global DHDFA. ${ }^{\mathrm{c}}$ The SCS fitting procedure led directly to the SOS parameters (see main text). ${ }^{\mathrm{d}}$ LC-DHDFA.

$\mathrm{MD}$, and error $\operatorname{span}\left(\Delta_{\mathrm{err}}\right)$. For example, PBEQIDH's SCS and SOS variants have identical RMSDs, MADs, and MDs and differ only in the error span. In this case, the SCS variant occupies a higher place in the ranking given its lower error span (0.78 vs. $0.81 \mathrm{eV})$. Most of the time these differences are negligible, but we decided to apply the same criteria throughout the present work to be consistent.

We note that the introduction of the SCS/SOS scheme is overall beneficial for each tested method when considering the statistics for the entire set of singlet-singlet excitations. Most methods display large reductions of their RMSD for both local-valence and Rydberg excitations upon introduction. For instance, the RMSD for RSX-QIDH for the complete set is reduced from $0.40 \mathrm{eV}$ to $0.21 / 0.24 \mathrm{eV}$ for its SCS/SOS variants, respectively. This coincides with a reduction of $0.25 \mathrm{eV}$ in the RMSD for local-valence excitations for both the SCS and SOS methods, and a reduction of $0.10 / 0.04$ $\mathrm{eV}$ for SCS/SOS for the Rydberg excitations. Some other methods, on the other hand, have an overall improved RMSD despite not improving for both local-valence and Rydberg excitations. For example, for B2PLYP the RMSD for local-valence excitations increases slightly from 0.24 to $0.27 \mathrm{eV}$ for its SCS/SOS variant. This is accompanied by a considerable reduction in the RMSD for the Rydberg excitations (from 0.53 to $0.30 \mathrm{eV}$ ), which leads to a reduction of the overall RMSD from 0.41 to $0.29 \mathrm{eV}$. We see something similar for PBE-QIDH were an improvement in the local-valence category compensated for slightly increased RMSDs for Rydberg states when SCS/SOS is applied.

When ranking all assessed methods according to their results for the entire set of singlet-singlet excitations, we find that PBEQIDH's SCS and SOS variants yield the lowest RMSDs with $0.16 \mathrm{eV}$ in both cases, closely followed by SCS- $\omega$ PBEPP86, SOS- $\omega$ B88PP86, and their related cousins SCS- $\omega$ B88PP86 and SOS- $\omega$ PBEPP86. These six methods are the only ones with RMSDs below $0.2 \mathrm{eV}$. We also identify four methods that share an RMSD of exactly $0.20 \mathrm{eV}$, namely, RS-PBE-P86, SCS- $\omega$ B2GP-PLYP, PBE-QIDH, and RS-PBE$\mathrm{PBE}$. It is noteworthy that the new RS-DHs, along with PBE-QIDH, still perform well despite being unscaled methods. In the same context, another well-performing unscaled DHDFA is $\omega \mathrm{B} 88 \mathrm{PP} 86$, which has the same RMSD of $0.21 \mathrm{eV}$ as SOS- $\omega$ B2GP-PLYP and SCS-RSXQIDH. Five DHDFAs immediately follow, all with the same RMSD of $0.22 \mathrm{eV}$, i.e., RS-B88LYP, SCS/SOS- $\omega$ B2PLYP, $\omega$ PBEPP86, SOSand SCS-B2GP-PLYP21. The remaining five DHDFAs with values below $0.3 \mathrm{eV}$ are SOSRSX-QIDH, the unscaled $\omega$ B2GP-PLYP, the related $\omega$ B2PLYP, B2GP-PLYP , and finally SCS/SOS-B2PLYP21. Lastly, RSX-QIDH and B2PLYP are the two worst performers for this set with RMSDs of 0.40 and $0.41 \mathrm{eV}$.

The MADs follow more or less the same trends as the RMSDs. It is remarkable that the SCS and SOS versions of PBE-QIDH, $\omega \mathrm{B} 88 \mathrm{PP} 86$, and $\omega \mathrm{PBEPP} 86$ are very close to the second definition of "chemical accuracy" for excited states, i.e., with average absolute deviations of up to $0.1 \mathrm{eV}$. On the other hand, the MDs show an interesting behavior once the SCS/SOS schemes are applied. Most of the tested unscaled methods, regardless of systematic over or underestimation of the excitation energies, show a systematic improve- 
Table 3: Statistical values (in eV) for all, local-valence, and Rydberg singlet-singlet excitations in the updated Gordon set using the TDA-DHDFA formalism. ${ }^{\text {a }}$

\begin{tabular}{|c|c|c|c|c|c|c|c|c|c|c|c|c|}
\hline \multirow[b]{2}{*}{ Method } & \multicolumn{4}{|c|}{ Overall } & \multicolumn{4}{|c|}{ Local-valence } & \multicolumn{4}{|c|}{ Rydberg } \\
\hline & MD & MAD & RMSD & $\Delta_{\text {err }}$ & MD & MAD & RMSD & $\Delta_{\text {err }}$ & MD & MAD & RMSD & $\Delta_{\text {err }}$ \\
\hline SCS-PBE-QIDH & 0.07 & 0.12 & 0.16 & 0.78 & 0.13 & 0.14 & 0.18 & 0.48 & 0.01 & 0.11 & 0.13 & 0.54 \\
\hline SOS-PBE-QIDH & 0.07 & 0.12 & 0.16 & 0.81 & 0.12 & 0.13 & 0.17 & 0.53 & 0.02 & 0.12 & 0.14 & 0.54 \\
\hline SCS- $\omega$ PBEPP 86 & 0.07 & 0.14 & 0.17 & 0.82 & 0.09 & 0.12 & 0.16 & 0.51 & 0.04 & 0.15 & 0.17 & 0.69 \\
\hline SOS- $\omega$ B88PP 86 & 0.05 & 0.14 & 0.18 & 0.94 & 0.11 & 0.13 & 0.17 & 0.54 & 0.00 & 0.16 & 0.19 & 0.79 \\
\hline SCS- $\omega$ B88PP86 & 0.08 & 0.15 & 0.19 & 0.95 & 0.15 & 0.16 & 0.19 & 0.53 & 0.01 & 0.15 & 0.18 & 0.77 \\
\hline SOS- $\omega$ PBEPP 86 & 0.10 & 0.16 & 0.19 & 0.81 & 0.10 & 0.13 & 0.19 & 0.57 & 0.10 & 0.18 & 0.20 & 0.73 \\
\hline RS-PBE-P86 & 0.04 & 0.14 & 0.20 & 0.88 & 0.13 & 0.18 & 0.24 & 0.86 & -0.05 & 0.10 & 0.14 & 0.72 \\
\hline SCS- $\omega$ B2GP-PLYP & 0.04 & 0.14 & 0.20 & 1.10 & 0.13 & 0.15 & 0.20 & 0.67 & -0.07 & 0.13 & 0.19 & 0.77 \\
\hline PBE-QIDH & 0.12 & 0.15 & 0.20 & 0.75 & 0.23 & 0.23 & 0.27 & 0.49 & 0.01 & 0.08 & 0.10 & 0.47 \\
\hline RS-PBE-PBE & 0.03 & 0.15 & 0.20 & 0.91 & 0.13 & 0.18 & 0.24 & 0.91 & -0.07 & 0.11 & 0.15 & 0.73 \\
\hline$\omega \mathrm{B} 88 \mathrm{PP} 86$ & 0.06 & 0.16 & 0.21 & 1.00 & 0.15 & 0.15 & 0.21 & 0.50 & -0.04 & 0.16 & 0.21 & 0.85 \\
\hline SOS- $\omega$ B2GP-PLYP & 0.06 & 0.16 & 0.21 & 1.11 & 0.15 & 0.17 & 0.23 & 0.70 & -0.04 & 0.14 & 0.19 & 0.79 \\
\hline SCS-RSX-QIDH & 0.12 & 0.17 & 0.21 & 0.83 & 0.14 & 0.15 & 0.21 & 0.57 & 0.10 & 0.19 & 0.21 & 0.73 \\
\hline RS-B88-LYP & -0.02 & 0.18 & 0.22 & 1.00 & 0.11 & 0.18 & 0.24 & 0.97 & -0.16 & 0.18 & 0.20 & 0.72 \\
\hline SCS/SOS- $\omega$ B2PLYP & 0.07 & 0.17 & 0.22 & 1.18 & 0.17 & 0.19 & 0.24 & 0.66 & -0.03 & 0.16 & 0.21 & 0.93 \\
\hline$\omega$ PBEPP86 & 0.13 & 0.18 & 0.22 & 0.94 & 0.17 & 0.17 & 0.23 & 0.58 & 0.08 & 0.19 & 0.22 & 0.80 \\
\hline SOS-B2GP-PLYP21 & -0.03 & 0.19 & 0.22 & 1.01 & 0.15 & 0.16 & 0.19 & 0.51 & -0.21 & 0.21 & 0.24 & 0.51 \\
\hline SCS-B2GP-PLYP21 & 0.01 & 0.19 & 0.22 & 1.00 & 0.19 & 0.20 & 0.23 & 0.50 & -0.19 & 0.19 & 0.22 & 0.52 \\
\hline SOS-RSX-QIDH & 0.12 & 0.20 & 0.24 & 0.83 & 0.08 & 0.16 & 0.21 & 0.64 & 0.16 & 0.24 & 0.27 & 0.80 \\
\hline$\omega \mathrm{B} 2 \mathrm{GP}-\mathrm{PLYP}$ & 0.12 & 0.19 & 0.25 & 1.08 & 0.26 & 0.26 & 0.32 & 0.65 & -0.03 & 0.11 & 0.15 & 0.73 \\
\hline$\omega \mathrm{B} 2 \mathrm{PLYP}$ & 0.12 & 0.20 & 0.26 & 1.14 & 0.26 & 0.26 & 0.32 & 0.62 & -0.03 & 0.14 & 0.18 & 0.89 \\
\hline B2GP-PLYP & -0.13 & 0.24 & 0.28 & 1.10 & 0.06 & 0.14 & 0.19 & 0.70 & -0.33 & 0.33 & 0.36 & 0.57 \\
\hline SCS/SOS-B2PLYP21 & -0.02 & 0.25 & 0.29 & 1.22 & 0.23 & 0.23 & 0.27 & 0.64 & -0.27 & 0.27 & 0.30 & 0.54 \\
\hline RSX-QIDH & 0.35 & 0.35 & 0.40 & 0.89 & 0.41 & 0.41 & 0.46 & 0.79 & 0.28 & 0.28 & 0.31 & 0.60 \\
\hline B2PLYP & -0.29 & 0.34 & 0.41 & 1.33 & -0.09 & 0.18 & 0.24 & 0.96 & -0.50 & 0.50 & 0.53 & 0.70 \\
\hline
\end{tabular}

a The aug-cc-pVTZ AO basis set was used in all cases. The DHDFAs are sorted by their overall RMSDs. The values for the RS-DH methods were taken from Ref. 80. The statistical estimators are Mean Deviation(MD),

Mean-Absolute Deviation (MAD), Root-Mean-Square Deviation (RMSD), and Error Span $\left(\Delta_{\text {err }}\right)$.

ment by having MDs that are closer to zero. For instance, RSX-QIDH overestimates the energies by on average $0.35 \mathrm{eV}$, which is improved by the SCS and SOS variants with $\mathrm{MD}=0.12 \mathrm{eV}$ in both cases. B2PLYP tends to underestimate the excitation energies $(\mathrm{MD}=-0.29 \mathrm{eV})$, whereas the $\mathrm{SCS} / \mathrm{SOS}$ B2PLYP21 variant has one of the best MDs with a value of only $-0.02 \mathrm{eV}$. Some unscaled DHDFAs with very good MDs that we would like to highlight are the three new $\mathrm{RS}-\mathrm{DH}$ methods (MDs range from 0.04 to $-0.02 \mathrm{eV}$ ) and $\omega \mathrm{B} 88 \mathrm{PP} 86(\mathrm{MD}=0.06 \mathrm{eV})$.

When focussing just on local-valence transitions, we observe similar trends as before. The best performer is SCS- $\omega$ PBEPP86 with $\mathrm{RMSD}=0.16 \mathrm{eV}$, which is closely followed by SOS- $\omega$ B88PP86 and PBE-QIDH. Other good performers below $0.2 \mathrm{eV}$ are SOS- $\omega$ PBEPP86, B2GP-PLYP and its SOS version, and SCS$\omega \mathrm{B} 88 \mathrm{PP} 86$. The majority of the remaining methods display results between $0.2 \mathrm{eV}$ and $0.3 \mathrm{eV}$, except for $\omega \mathrm{B} 2 \mathrm{PLYP}, \omega \mathrm{B} 2 \mathrm{GP}-\mathrm{PLYP}$ (both with RMSDs of $0.32 \mathrm{eV}$ ), and RSX-QIDH $(0.46 \mathrm{eV})$.

We end the discussion of singlet excitations by analyzing the Rydberg category. The unscaled PBE-QIDH functional is the best performer with an excellent value of $0.10 \mathrm{eV}$.
What is surprising is the fact that its SCS and SOS variants display slightly larger RMSDs of $0.13 \mathrm{eV}$ and $0.14 \mathrm{eV}$, respectively. However, as we mentioned before, this mishap is greatly compensated for by its reduction of about $0.10 \mathrm{eV}$ in the local-valence category. The RS-PBE-P86 method has a value of $0.14 \mathrm{eV}$ and occupies the third place in the ranking, followed by RS-PBE-PBE and $\omega$ B2GP-PLYP with RMSDs of $0.15 \mathrm{eV}$. The next DHDFAs in our ranking are SCS- $\omega$ PBEPP86, $\omega$ B2PLYP, SCS- $\omega$ B88PP86, SCS- and SOS- $\omega$ B2GP-PLYP, and SOS- $\omega$ B88PP86. The remaining methods have RMSDs between $0.20 \mathrm{eV}$ (RS-B88-LYP and SOS- $\omega$ PBEPP86) and $0.53 \mathrm{eV}$ (B2PLYP).

\subsubsection{Analysis of Vertical Singlet- Triplet Excitations}

Following the same idea as presented by us in Ref. 41, we perform two different analyses for singlet-triplet excitations. The first type covers all transitions between the ground state singlet to the various triplet excited states, which we denote as $\mathrm{S} 0-\mathrm{TN}$, with $\mathrm{N} \geq 1$. The second type focuses on the energy splitting between the first singlet and triplet excited states, denoted as S1-T1. The latter is particularly intriguing for potential future applications of well-performing methods in areas such as singlet-fission materi- 
Table 4: Statistical values (in eV) for the S0-TN transitions in the updated Gordon set using the TDA-DHDFA formalism. ${ }^{\text {a }}$

\begin{tabular}{|c|c|c|c|c|c|c|c|c|c|c|c|c|}
\hline \multirow[b]{2}{*}{ Method } & \multicolumn{4}{|c|}{ Overall } & \multicolumn{4}{|c|}{ Local-valence } & \multicolumn{4}{|c|}{ Rydberg } \\
\hline & MD & MAD & RMSD & $\Delta_{\text {err }}$ & MD & MAD & RMSD & $\Delta_{\text {err }}$ & MD & MAD & RMSD & $\Delta_{\text {err }}$ \\
\hline SCS-PBE-QIDH & -0.04 & 0.06 & 0.08 & 0.31 & -0.04 & 0.06 & 0.08 & 0.30 & -0.04 & 0.05 & 0.06 & 0.15 \\
\hline SOS-PBE-QIDH & -0.04 & 0.07 & 0.08 & 0.33 & -0.04 & 0.06 & 0.08 & 0.31 & -0.04 & 0.08 & 0.10 & 0.30 \\
\hline PBE-QIDH & -0.04 & 0.07 & 0.09 & 0.37 & 0.03 & 0.07 & 0.09 & 0.41 & -0.03 & 0.08 & 0.10 & 0.33 \\
\hline SCS- $\omega$ B 88 PP 86 & -0.07 & 0.09 & 0.12 & 0.40 & -0.03 & 0.07 & 0.10 & 0.35 & -0.03 & 0.11 & 0.12 & 0.36 \\
\hline SOS- $\omega$ PBEPP 86 & -0.05 & 0.10 & 0.12 & 0.47 & -0.06 & 0.08 & 0.10 & 0.37 & 0.02 & 0.11 & 0.13 & 0.42 \\
\hline$\omega$ РBEPP 86 & 0.00 & 0.10 & 0.12 & 0.37 & -0.04 & 0.08 & 0.10 & 0.37 & -0.02 & 0.12 & 0.13 & 0.35 \\
\hline SOS- $\omega$ B 88 PP 86 & -0.09 & 0.10 & 0.13 & 0.43 & -0.03 & 0.08 & 0.10 & 0.34 & -0.04 & 0.14 & 0.14 & 0.37 \\
\hline SOS- $\omega$ B2GP-PLYP & -0.05 & 0.10 & 0.13 & 0.60 & 0.01 & 0.08 & 0.10 & 0.33 & -0.09 & 0.12 & 0.15 & 0.40 \\
\hline$\omega B 2 G P-P L Y P$ & -0.02 & 0.11 & 0.13 & 0.58 & -0.02 & 0.09 & 0.10 & 0.48 & -0.13 & 0.13 & 0.15 & 0.28 \\
\hline SCS- $\omega$ B2GP-PLYP & -0.08 & 0.11 & 0.14 & 0.56 & -0.08 & 0.09 & 0.11 & 0.35 & 0.04 & 0.14 & 0.16 & 0.49 \\
\hline$\omega \mathrm{B} 88 \mathrm{PP} 86$ & -0.07 & 0.11 & 0.14 & 0.45 & -0.08 & 0.09 & 0.11 & 0.37 & -0.10 & 0.14 & 0.17 & 0.43 \\
\hline SCS- $\omega$ PBEPP 86 & -0.09 & 0.12 & 0.14 & 0.44 & -0.05 & 0.09 & 0.11 & 0.45 & -0.13 & 0.14 & 0.18 & 0.41 \\
\hline SCS/SOS- $\omega$ B2PLYP & -0.08 & 0.11 & 0.15 & 0.62 & -0.04 & 0.09 & 0.11 & 0.48 & -0.16 & 0.16 & 0.19 & 0.38 \\
\hline SOS-B2GP-PLYP21 & -0.04 & 0.11 & 0.15 & 0.64 & 0.06 & 0.09 & 0.11 & 0.43 & 0.18 & 0.18 & 0.20 & 0.22 \\
\hline$\omega \mathrm{B} 2 \mathrm{PLYP}$ & -0.06 & 0.12 & 0.15 & 0.61 & -0.02 & 0.10 & 0.12 & 0.51 & -0.16 & 0.16 & 0.21 & 0.40 \\
\hline SCS-B2GP-PLYP21 & -0.01 & 0.12 & 0.15 & 0.65 & 0.02 & 0.10 & 0.12 & 0.51 & -0.19 & 0.19 & 0.21 & 0.28 \\
\hline SCS-RSX-QIDH & -0.10 & 0.13 & 0.15 & 0.49 & 0.09 & 0.11 & 0.13 & 0.42 & -0.16 & 0.17 & 0.22 & 0.49 \\
\hline SOS-RSX-QIDH & -0.07 & 0.13 & 0.15 & 0.57 & -0.12 & 0.12 & 0.14 & 0.30 & -0.17 & 0.18 & 0.22 & 0.38 \\
\hline RSX-QIDH & 0.06 & 0.15 & 0.17 & 0.57 & -0.12 & 0.13 & 0.15 & 0.36 & -0.22 & 0.22 & 0.23 & 0.28 \\
\hline SCS/SOS-B2PLYP21 & -0.02 & 0.16 & 0.19 & 0.71 & -0.14 & 0.14 & 0.15 & 0.29 & -0.28 & 0.28 & 0.30 & 0.32 \\
\hline B2GP-PLYP & -0.13 & 0.16 & 0.22 & 0.62 & 0.01 & 0.13 & 0.16 & 0.57 & -0.37 & 0.37 & 0.38 & 0.27 \\
\hline B2PLYP & -0.24 & 0.26 & 0.34 & 0.87 & -0.11 & 0.14 & 0.17 & 0.45 & -0.56 & 0.56 & 0.57 & 0.39 \\
\hline
\end{tabular}

a The aug-cc-pVTZ AO basis set was used in all cases. The DHDFAs are sorted by their overall RMSDs. The statistical estimators are Mean Deviation(MD), Mean-Absolute Deviation (MAD), Root-Mean-Square Deviation $(\mathrm{RMSD})$, and Error Span $\left(\Delta_{\mathrm{err}}\right)$.

als. $\frac{107}{111}$ It also gives us first insights into the robustness of a method, as there is no guarantee that a method that performs well for singlet excitations is also suitable for triplet ones.

\section{All Singlet-Triplet Excitations in this set (S0-TN)}

In Table 4, we see that nearly every tested TDA-DHDFA seems to describe singlet-triplet excitations with a very good or acceptable RMSD below $0.2 \mathrm{eV}$, except for B2PLYP $(0.34 \mathrm{eV})$ and B2GP-PLYP $(0.22 \mathrm{eV})$. PBEQIDH and its SCS/SOS variants perform outstandingly well, with RMSDs below the second definition of "chemical accuracy" for excitation energies, with values of $0.08-0.09 \mathrm{eV}$. Just a few hundredths of an eV above, we locate SCS- $\omega$ B88PP86, SOS- $\omega$ PBEPP86, and the unscaled $\omega$ PBEPP86 $(\mathrm{RMSD}=0.12 \mathrm{eV})$, which are closely followed by SOS- $\omega$ B88PP86, SOS- $\omega$ B2GP-PLYP, and the unscaled $\omega$ B2GPPLYP $($ RMSD $=0.13 \mathrm{eV})$. We then find that nine DHDFAs, both scaled and unscaled ones, present good values between $0.14-0.15 \mathrm{eV}$, and based on our other criteria outlined earlier we rank them from SCS- $\omega$ B2GP-PLYP to SOS-RSX-QIDH. Interestingly, RSX-QIDH and SCS/SOS-B2PLYP21 deliver acceptable results below $0.20 \mathrm{eV}$. We also notice that, in general, most unscaled TDA-DHDFAs are slightly outperformed by their corresponding SCS/SOS variants.

The MDs display small underestimations with absolute MDs below $0.1 \mathrm{eV}$, except for B2PLYP $(-0.24 \mathrm{eV})$ and B2GP-PLYP $(-0.13 \mathrm{eV})$. We point out that the only DHDFA that displays an overestimation is RSX-QIDH $(0.06 \mathrm{eV})$. In general, inclusion of SCS/SOS seems to keep the MDs nearly unchanged, such as in PBEQIDH, or it produces a slight red shift, such as in wPBEPP86 (see Table 4.2.3)

\section{S1-T1 Analysis}

In Table 5, we see that the statistics for the S1T1 splitting show similar trends as for the S0TN analysis with most RMSDs being below 0.2 $\mathrm{eV}$. In the updated Gordon set, most low-lying excitation energies belong to the local-valence category, and only for the water molecule we have to analyze a Rydberg excitation.

B2GP-PLYP's SOS and SCS variants are at the top of the ranking with RMSDs of 0.08 and $0.09 \mathrm{eV}$, respectively. PBE-QIDH, its SCS variant, and $\omega$ B2GP-PLYP's SCS and SOS variants also display the same outstanding result with RMSD $=0.1 \mathrm{eV}$. Nine methods follow closely ranging from the unscaled B2GP-PLYP (0.12 $\mathrm{eV})$ to the scaled SCS- $\omega$ PBEPP86 $(0.15 \mathrm{eV})$. Other acceptably well-performing DHDFAs are B2PLYP, $\omega B 2$ GP-PLYP, SOS-RSX-QIDH, and 
PBE-QIDH, and $\omega B 2 P L Y P$. Finally, SCS-RSXQIDH and its unscaled variant conclude the list with values of 0.22 and $0.33 \mathrm{eV}$, respectively.

One major difference to the previous S0-TN analysis is the fact that the MDs are mostly positive, with only one exception (B2PLYP). The reason is simply that most DHDFAs display a systematic overestimation for singletsinglet excitations, whereas they systematically underestimate singlet-triplet excitations. As a result, it is expected that the S1-T1 energy splitting also displays an overestimation. 41

Our findings for the updated Gordon set are very promising. The SCS/SOS variants show considerable improvements over their unscaled parents for singlet-singlet transitions, while for singlet-triplet excitations there is a more modest improvement. In sections 5 and 6 we will further discuss how our new SCS and SOS variants perform in an extensive crossvalidation study that covers both singlet-singlet and singlet-triplet excitations, including CT excitations, which have not been part of our parameter training.

Table 5: Statistical values (in eV) for the S1-T1 energy splitting on the updated Gordon set using the TDA-DHDFA formalism. ${ }^{\mathrm{a}}$

\begin{tabular}{|c|c|c|c|c|}
\hline Method & MD & MAD & RMSD & $\Delta_{\text {err }}$ \\
\hline SOS-B2GP-PLYP21 & 0.02 & 0.07 & 0.08 & 0.26 \\
\hline SCS-B2GP-PLYP21 & 0.03 & 0.08 & 0.09 & 0.29 \\
\hline SOS-PBE-QIDH & 0.07 & 0.09 & 0.10 & 0.26 \\
\hline SOS- $\omega$ B2GP-PLYP & 0.07 & 0.09 & 0.10 & 0.26 \\
\hline SCS- $\omega$ B2GP-PLYP & 0.08 & 0.09 & 0.10 & 0.24 \\
\hline SCS-PBE-QIDH & 0.08 & 0.09 & 0.10 & 0.23 \\
\hline B2GP-PLYP & 0.01 & 0.10 & 0.12 & 0.40 \\
\hline$\omega$ PBEPP86 & 0.09 & 0.11 & 0.13 & 0.28 \\
\hline SCS/SOS- $\omega$ B 2 PLYP & 0.10 & 0.12 & 0.13 & 0.27 \\
\hline SOS- $\omega \mathrm{B} 88 \mathrm{PP} 86$ & 0.10 & 0.12 & 0.13 & 0.35 \\
\hline$\omega \mathrm{B} 88 \mathrm{PP} 86$ & 0.11 & 0.12 & 0.14 & 0.33 \\
\hline SCS- $\omega$ B88PP86 & 0.11 & 0.13 & 0.15 & 0.33 \\
\hline SCS/SOS-B2PLYP21 & 0.04 & 0.12 & 0.15 & 0.47 \\
\hline SOS- $\omega$ PBEPP86 & 0.09 & 0.11 & 0.15 & 0.44 \\
\hline SCS- $\omega$ PBEPP 86 & 0.11 & 0.13 & 0.15 & 0.38 \\
\hline B2PLYP & -0.03 & 0.13 & 0.16 & 0.50 \\
\hline$\omega \mathrm{B} 2 \mathrm{GP}-\mathrm{PLYP}$ & 0.14 & 0.15 & 0.18 & 0.41 \\
\hline SOS-RSX-QIDH & 0.11 & 0.13 & 0.19 & 0.54 \\
\hline PBE-QIDH & 0.16 & 0.16 & 0.19 & 0.41 \\
\hline$\omega \mathrm{B} 2 \mathrm{PLYP}$ & 0.16 & 0.17 & 0.20 & 0.44 \\
\hline SCS-RSX-QIDH & 0.18 & 0.18 & 0.22 & 0.42 \\
\hline RSX-QIDH & 0.28 & 0.28 & 0.33 & 0.56 \\
\hline
\end{tabular}

a The aug-cc-pVTZ AO basis set was used in all cases. The DHDFAs are sorted by their overall RMSDs. The statistical estimators are Mean Deviation(MD), Mean-Absolute Deviation (MAD), Root-Mean-Square Deviation (RMSD), and Error Span $\left(\Delta_{\text {err }}\right)$.
5 Cross-Validation for Vertical Singlet-Singlet Excitations

\subsection{The $\omega$ B2(GP-)PLYP Training Set}

The $\omega \mathrm{B} 2(\mathrm{GP}-)$ PLYP "training set"40 is a slight modification of the original set used by Schwabe and Goerigk for their parametrization of TD(A)-SCS/SOS-DHDFAs in 2017. ${ }^{39} \mathrm{It}$ contains 34 vertical singlet-singlet excitations, made of up 28 valence and 6 Rydberg transitions. The reference values are based on Coupled Cluster Singles Doubles with Perturbative Triples Excitation Correction 112 [CCSDR(3)] with the large aug-cc-pVTZ AO basis set. Note that herein we do not use this set for any fitting procedure but called it "training set" to indicate that it was used during the development of our $\omega$ B2(GP-)PLYP functionals. $\frac{40}{}$ Herein, we present the RMSD and MAD values for our herein assessed TDA-DHDFAs in Fig. 1, where the results are, once again, broken down into local-valence and Rydberg transitions. The methods are sorted according to their RMSD values for the overall set, which are also shown on top of the bars. All raw as well as other statistical data are provided in the Supporting Information. We note in passing that the "top 10" methods are SCS/SOS variants, with the RS-DH methods coming right after them. Indeed, the RS-DH methods have previously been among the most accurate DHDFAs in Ref. 80 and it was argued that it was due to range-separation in the perturbative CIS(D) treatment. We show here nevertheless that even better results can be achieved through the SCS/SOS technique without the need of introducing range-separation in the perturbative treatment. This is an encouraging outcome and a strong indicator that the SCS/SOS methods can be transferred to systems other than the ones used in the fit. We now proceed with the analysis of the RMSDs for this set.

PBE-QIDH's SOS and SCS variants are at the top of the ranking with equal RMSDs of $0.12 \mathrm{eV}$, but are closely followed by SCS- 


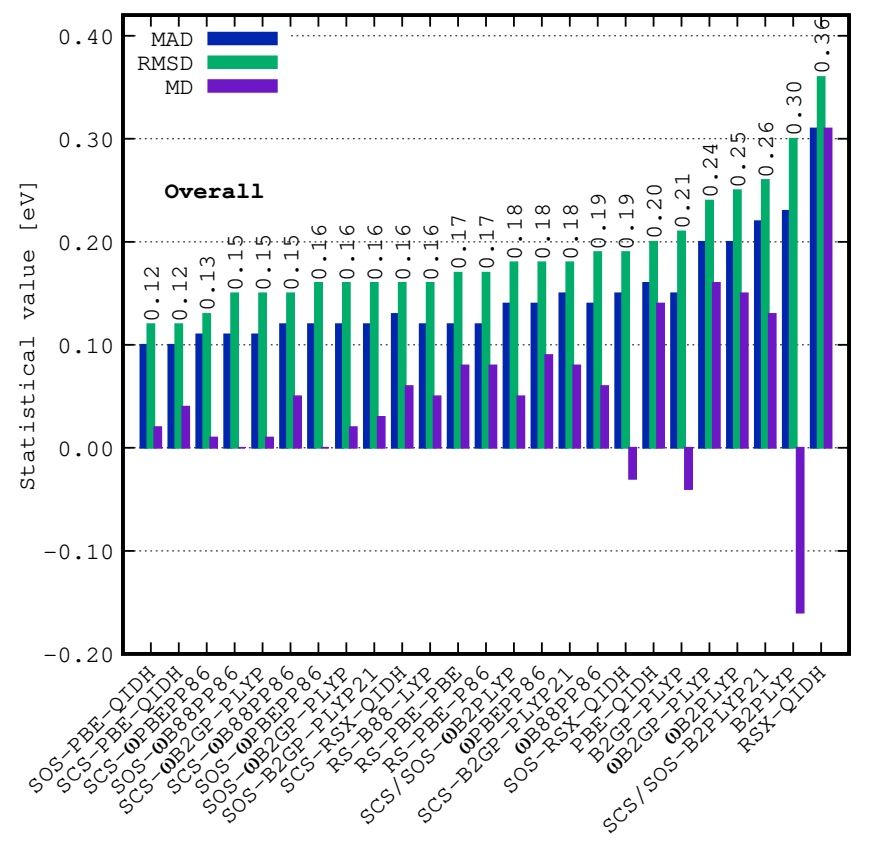

Figure 1: Statistical values (in eV) for the $\omega \mathrm{B} 2(\mathrm{GP}-$ )PLYP training set, namely RMSDs (values shown over each bar), MADs, and MDs. The values for the RS-DH methods were taken from Ref. 80 The aug-cc-pVTZ AO basis set was used in all cases.

wPBEPP86 with just one-hundredth of an eV above the former two. Next, $\omega B 88$ PP86's SCS and SOS variants along with SCS- $\omega$ B2GPPLYP display the same RMSD, followed by a group of five methods all with identical RMSDs: SOS- $\omega$ PBEPP86, SOS- $\omega$ B2GP-PLYP, SOSB2GP-PLYP21, SCS-RSX-QIDH, and RSB88-LYP. RS-PBE-PBE and RS-PBE-P86 are placed slightly above those $(\mathrm{RMSD}=0.17 \mathrm{eV})$ and they are followed by SCS/SOS- $\omega$ B2PLYP, $\omega$ PBEPP86, and SCS-B2GP-PLYP21 (RMSD $=0.18 \mathrm{eV})$. The remaining two DHDFAs below an RMSD of $0.2 \mathrm{eV}$ are $\omega \mathrm{B} 88 \mathrm{PP} 86$ and SOSRSX-QIDH. Thus, we have identified a total of 18 methods that yield RMSDs below $0.2 \mathrm{eV}$ most of which are SCS or SOS variants, with SCS/SOS-B2PLYP21 being the only exception $(\mathrm{RMSD}=0.26 \mathrm{eV})$. All remaining methods have RMSDs that range from $0.20 \mathrm{eV}$ (PBE-QIDH) to $0.36 \mathrm{eV}$ (RSX-QIDH).

Our new SCS and SOS variants also display improvements when just analyzing localvalence transitions. We briefly summarize that there are four methods that occupy the first position in the ranking and also fulfill the second definition of "chemical accuracy" given above, namely, SOS- $\omega$ B88PP86, SOS-PBE-QIDH, and $\omega B 2 G P-P L Y P ' s$ SCS and SOS variants with RMSDs of $0.10 \mathrm{eV}$. Almost every SCS and SOS method is ranked in the best places and performs better than its unscaled parent, except for SCS-B2GP-PLYP21 and SCS/SOSB2PLYP21. For Rydberg transitions, some of the SCS and SOS variants have slightly increased RMSDs compared to their unscaled parents. PBE-QIDH is such an example where the RMSD increases by 0.04 and $0.06 \mathrm{eV}$ when the SCS and SOS formalisms are applied.

In the following sections, we will test our new methods on more challenging systems where unscaled DHDFAs have presented problems in the past. 404180

\subsection{The Loos and Jacquemin Benchmark Set}

We also analyzed the systems introduced by Loos, Jacquemin, and co-workers in their 2018 benchmark set.113 However, similarly to our previous works, 4041 and due to overlap with already tested systems, we only consider 29 of their excitations divided into 16 valence and 13 Rydberg transitions in 11 different systems, namely acetaldehyde, acetylene, carbon monoxide, diazomethane, dinitrogen, hydrogen chloride, hydrogen sulfide, ketene, methanimine, nitrosomethane, and thioformaldehyde. All reference values have CC3/aug-cc-pVTZ quality. All statistical results are shown in Fig. 2.

The best RMSD for this set is obtained with SOS-B2GP-PLYP21 (0.14 eV), closely followed by the unscaled B2GP-PLYP, SCS$\omega B 2 G$-PLYP, and SOS- $\omega$ B88PP86 (RMSD $=0.15 \mathrm{eV}$ in each case). We also highlight SOS- $\omega$ B2GP-PLYP, SCS-B2GP-PLYP21, $\omega \mathrm{B} 88 \mathrm{PP} 86$, and SCS- $\omega \mathrm{B} 88 \mathrm{PP} 86$ (RMSD = $0.16 \mathrm{eV}$ in each case). The last three methods with an RMSD below $0.2 \mathrm{eV}$ are SCS/SOS$\omega B 2 P L Y P$, SCS/SOS-B2PLYP21 and SOSPBE-QIDH. The four DHDFAs with RMSDs of exactly $0.2 \mathrm{eV}$ are $\omega \mathrm{B} 2 \mathrm{PLYP}$, SCS-PBEQIDH, and wPBEPP86's SCS and SOS variants. Most of the remaining methods with RMSDs above $0.2 \mathrm{eV}$ are unscaled DHDFAs, such as $\omega$ B2GP-PLYP, the three RS-DH meth- 


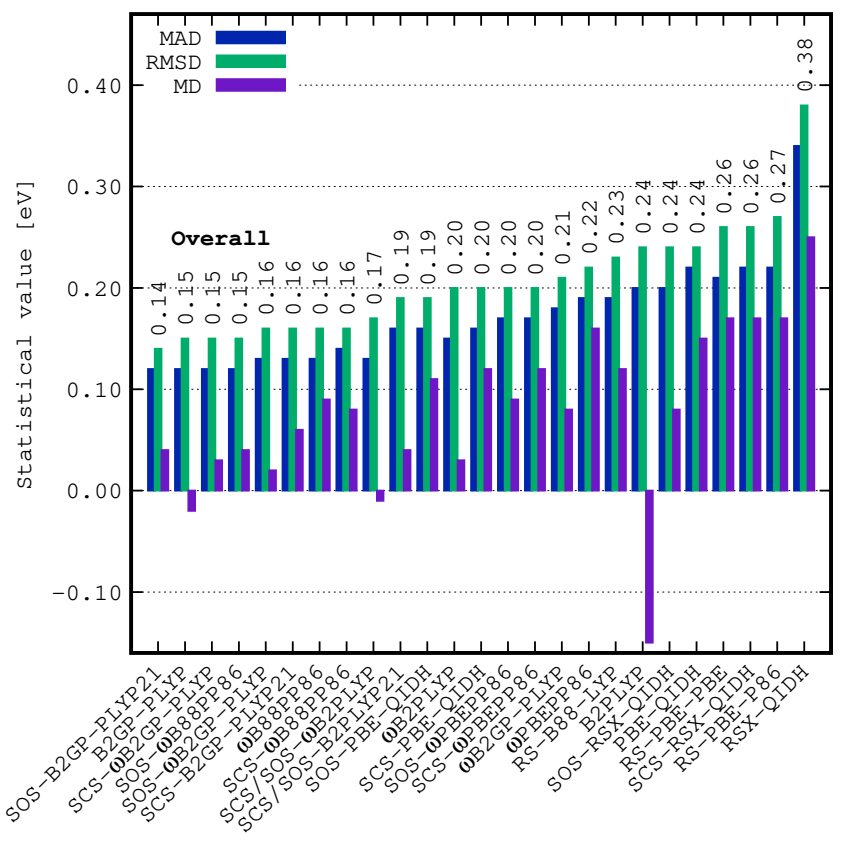

Figure 2: Statistical values (in eV) for the Loos and Jacquemin set, namely RMSDs (values shown over each bar), MADs, and MDs. The values for the RS-DH methods were taken from Ref. 80 The aug-cc-pVTZ AO basis set was used in all cases.

ods, or PBE-QIDH. That being said, we find that RSX-QIDH's SCS and SOS variants also belong to this range. The unscaled RSX-QIDH yields the highest RMSD of $0.38 \mathrm{eV}$, confirming other studies that this LC-DHDFA is often less accurate than its global PBE-QIDH counterpart. $32 \mid 41$

When focussing solely on local-valence excitations, we notice similar trends as for the two previously discussed sets with most of the SCS and SOS variants being positioned at the top and RMSDs ranging from 0.13 $\mathrm{eV}$ (SOS- $\omega$ PBEPP86 and SOS-PBE-QIDH) to $0.16 \mathrm{eV}$ (SOS-RSX-QIDH, SCS- $\omega$ B2GP-PLYP, and $\omega \mathrm{B} 88 \mathrm{PP} 86)$. Note that the RS-DH methods perform equally well with RMSDs of 0.16-0.17 eV, values that they share with B2PLYP, B2GP-PLYP, SCS-RSX-QIDH, SOSB2GP-PLYP21, and SOS- $\omega$ B2GP-PLYP. The last two methods with RMSDs below $0.2 \mathrm{eV}$ are $\omega$ PBEPP86 and SCS-B2GP-PLYP21 (0.18 $\mathrm{eV})$. All remaining methods have RMSDs above $0.20 \mathrm{eV}$.

As we already pointed out in Ref. 40, we called this set the difficult cases set because its Rydberg states do not seem to require the LC scheme, contrary to the examples discussed earlier. Note that this trend is also seen for the RS-DH methods, which show large blue-shifts for this category (RMSD $=0.30$ and $0.35 \mathrm{eV}$ for RS-B88-LYP and RS-PBE$\mathrm{P} 86$, respectively). Hence, it is not surprising that the two top places in our ranking are global DHDFAs methods (SOS-B2GP-PLYP21 and SCS/SOS-B2PLYP21). However, our new SCS- and SOS-LC-DHDFAs improve over their unscaled parents and yield more accurate results, for instance, SCS/SOS- $\omega$ B2PLYP and $\omega B 2 P L Y P(0.12$ vs. $0.14 \mathrm{eV})$, SCS- $\omega$ B2GPPLYP and $\omega B 2 G P-P L Y P(0.14$ vs. $0.20 \mathrm{eV}$ ).

In summary, our SCS/SOS variants of global and LC-DHDFAs are the best-performing methods even for the difficult cases presented by Loos, Jacquemin, and co-workers. None of these systems were assessed in the previous sets, demonstrating that our new methods are robust enough, even for the treatment of challenging systems like the ones discussed in this section.

\subsection{The "Exotic-Molecules" Set}

In mid-2020, Loos and Jacquemin et al. presented a new benchmark set under the name "exotic molecules set". With that name the authors intended to imply that each of the molecules in this set includes at least one atom representing elements that is uncommon in usual organic-molecule test sets, namely: F, $\mathrm{Cl}$, Si, or P.114 The overall set comprises 19 local-valence singlet-singlet excitations from 14 different systems with high-level CC3/aug-ccpVTZ reference values. We remind the reader that the inclusion of these "exotic molecules" made the TD-DHDFA scheme unable to correctly describe these kind of systems and better results were obtained with the TDA-DHDFA formalism, as we thoroughly discussed in Ref. 41. Therefore, the user might take this into consideration when the system under study presents any of the aforementioned species.

Based on the RMSDs presented in Fig. 3, the RS-DH methods yield the lowest RMSDs $(<0.1$ $\mathrm{eV}$ ), which is an outstanding finding. B2GPPLYP also displays an excellent value of exactly 


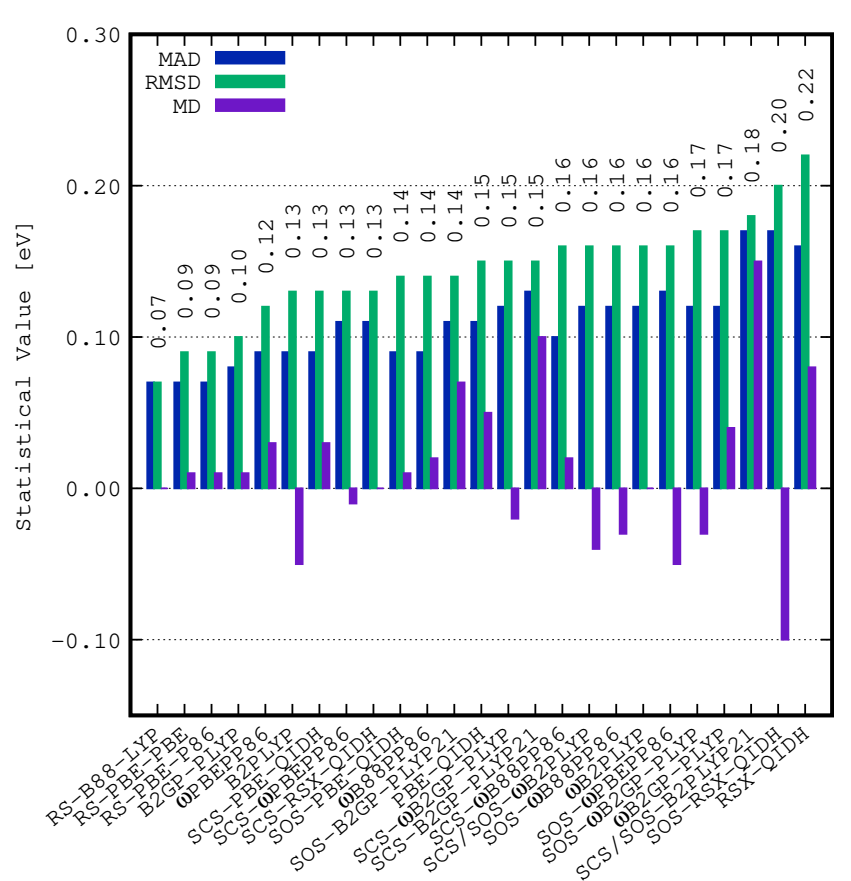

Figure 3: Statistical values (in eV) for the "exotic-molecules" set, namely RMSDs (values shown over each bar), MADs, and MDs. The values for the RS-DH methods were taken from Ref. 80 The aug-cc-pVTZ AO basis set was used in all cases.

$0.10 \mathrm{eV}$ for this test set, closely followed by our new $\omega$ PBEPP86 (0.12 eV) and B2PLYP (0.13 $\mathrm{eV})$. Our new SCS and SOS methods feature in the ranking from this point onward, with SCSPBE-QIDH, SCS- $\omega$ PBEPP86, and SCS-RSXQIDH being the first ones with good values of $0.13 \mathrm{eV} .14$ other methods, all of which developed for this study, have RMSDs that range from 0.14 to $0.18 \mathrm{eV}$. Lastly, SOS-RSX-QIDH and its parent RSX-QIDH are placed in last position with RMSDs of 0.20 and $0.22 \mathrm{eV}$. respectively. We conclude that almost every assessed method performs fairly well compared to the previously sets given that their RMSDs mostly lie below $0.20 \mathrm{eV}$.

In general, the MDs are well-balanced for this set, regardless of the use of SCS or SOS. Except for SCS/SOS-B2PLYP21 and SCS-B2GPPLYP21 $(\mathrm{MDs}=0.15 \mathrm{eV})$, and SOS-RSX-QIDH $(\mathrm{MD}=-0.10 \mathrm{eV})$, every other method has absolute MDs below $0.1 \mathrm{eV}$.

\subsection{The Thiel Benchmark Set}

To keep investigating our new SCS/SOS methods' capabilities a bit further, we also analyze parts of the popular benchmark set intro- duced by Thiel and co-workers. 27 The original set comprises 167 valence excitations, 104 of which are singlet-singlet excitations with various WFT reference values, out of which we chose the CC3/TZVP115 ones in agreement with previous works in Refs. 39,41. In order to avoid duplication with previous sets, we only analyze 36 local-valence singlet excitations from 8 different systems, namely: acetamide, benzoquinone, imidazole, norbornadiene, octatetraene, propanamide, pyridazine, and pyrimidine.

This test set differs from previous ones given the large RMSDs for every tested method. After a closer inspection, we found that the $2^{1} \mathrm{Ag}_{\mathrm{g}}$ state in octatetraene displays a very large overestimation, reaching more than $1 \mathrm{eV}$ in some cases. In 2011, Angeli and Pastore ${ }^{116}$ considered octatetraene as a somewhat complicated system due to its high double-excitation character. Previous work on TD-B2(GP-)PLYP also showed this state to be problematic. ${ }^{28}$ In light of this finding, we report the statistical results by considering and excluding this state, where the latter case is shown in parentheses (Fig. 4).

When considering all excitations, PBEQIDH's SCS and SOS variants are in the lead, closely followed by the unscaled B2GP-PLYP and its SOS variant, all of them with RMSDs slightly above $0.2 \mathrm{eV}$. Seven DHDFAs have RMSDs between 0.26 and $0.29 \mathrm{eV}$; with the exception of PBE-QIDH, all methods have been newly developed for this study. Five methods yield high RMSDs that range from 0.33 to $0.36 \mathrm{eV}$, namely, SCS/SOS- $\omega$ B2PLYP, RSXQIDH's SCS and SOS variants, and $\omega B 2 G P-$ PLYP's SCS and SOS variants. Finally, the RS-DH methods and the unscaled $\omega$ B2PLYP have values of $0.40 \mathrm{eV}$, whereas $\omega \mathrm{B} 2 \mathrm{GP}-\mathrm{PLYP}$ and RSX-QIDH come last with RMSDs of 0.43 , and $0.54 \mathrm{eV}$, respectively.

When excluding the $2^{1} \mathrm{~A}_{\mathrm{g}}$ state in octatetraene, the ranking of methods changes. Now, most of the SCS and SOS variants are placed at the top. Compared to the analysis in the previous paragraph, those methods with the highest RMSDs benefit from a large reduction (on average $0.1 \mathrm{eV}$ ), whereas the ones with the low RMSDs benefit from a smaller 


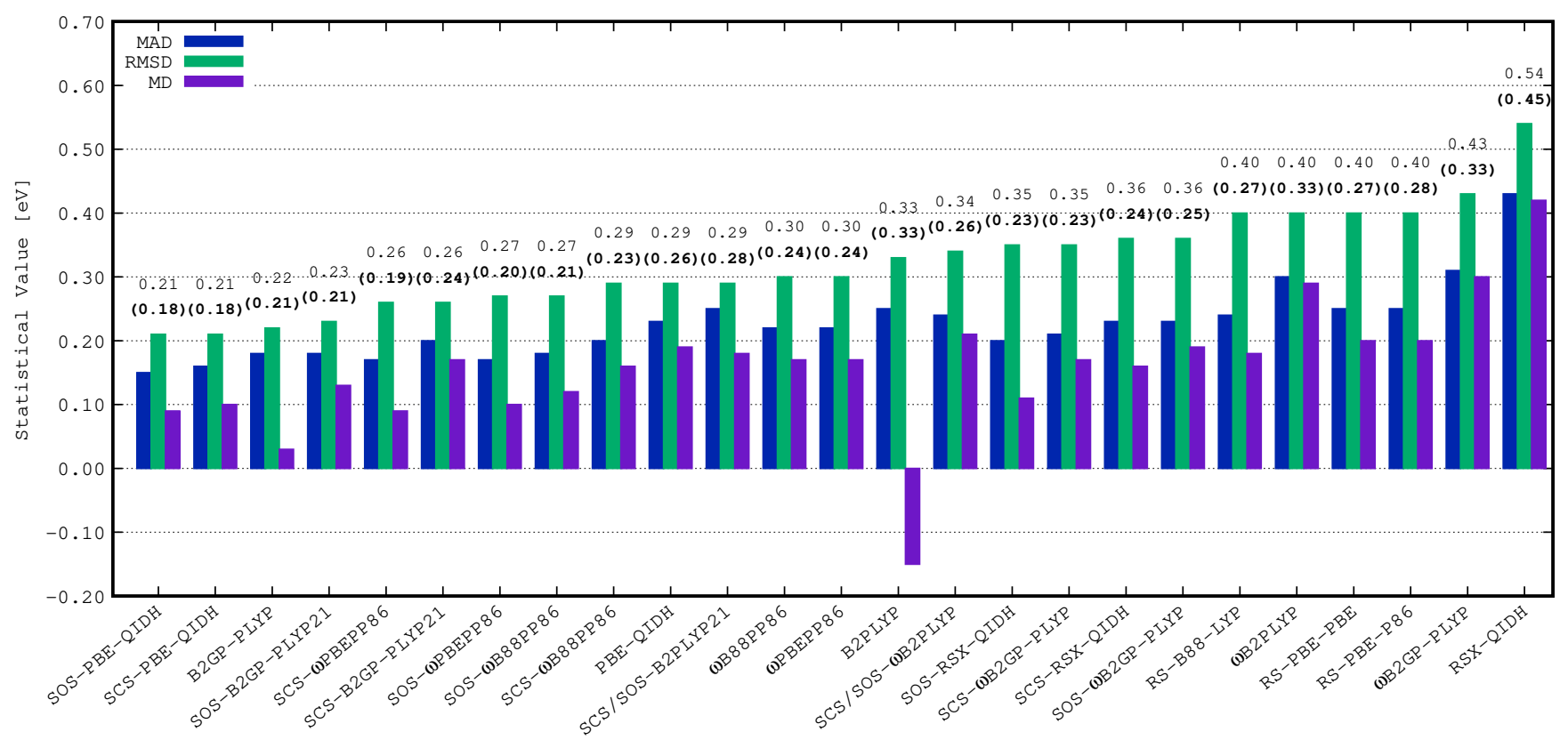

Figure 4: Statistical values (in eV) for the Thiel set, namely RMSDs (values shown over each bar), MADs, and MDs. Values in parentheses are RMSDs after excluding the $2^{1} \mathrm{~A}_{\mathrm{g}}$ state in octatetraene. The values for the RS-DH methods were taken from Ref. [80. The TZVP AO basis set was used in all cases.

reduction (on average $0.05 \mathrm{eV}$ ). The three best performers this time are PBE-QIDH's SCS and SOS variants and SCS- $\omega$ PBEPP86 with RMSDs below $0.2 \mathrm{eV}$. We also see improvements for various methods after excluding the $2^{1} \mathrm{~A}_{\mathrm{g}}$ state in octatetraene, with reductions in the RMSDs of up to $0.13 \mathrm{eV}$, such as SCS- $\omega$ B2GP-PLYP, SCS/SOS- $\omega$ B2PLYP, or the three RS-DH methods. They now all perform similarly to unscaled PBE-QIDH. Lastly, the unscaled B2PLYP, $\omega$ B2PLYP, and $\omega$ B2GP$\mathrm{PLYP}$ also have improved values $(\mathrm{RMSD}=0.33$ $\mathrm{eV}$ in each case), whereas RSX-QIDH is still in last place with a value of $0.45 \mathrm{eV}$. Overall, we can conclude how most SCS/SOS variants show superior performance for a set that seems to be challenging to describe.

\section{5 ${ }^{1} \mathrm{~L}_{\mathrm{a}}$ and ${ }^{1} \mathrm{~L}_{\mathrm{b}}$ Transitions in Linear Polycyclic Aromatic Hydrocarbons}

Linear polycyclic aromatic hydrocarbons (PAHs) have gained particular interest due to their possible applications in light-emitting diodes or photovoltaics, to name two examples. According to the Platt nomenclature, the two lowest singlet-singlet excitations are usually called ${ }^{1} \mathrm{~L}_{\mathrm{a}}$ and ${ }^{1} \mathrm{~L}_{\mathrm{b}} \cdot 117$ The former exhibits a polarization along the short axis of the molecule, whereas the second is polarized along the long axis. $\frac{118}{10}$ It has also been suggested that the ${ }^{1} \mathrm{~L}_{\mathrm{a}}$ state has ionic character, while the ${ }^{1} \mathrm{~L}_{\mathrm{b}}$ state has covalent character.119 121 Conventional TD-DFT fails to describe both states with comparable accuracy; as shown in detail in Refs. 35118 and 40, a good description of one state comes at the cost of the other. In summary, LC-hybrid functionals provide a better description of ${ }^{1} \mathrm{~L}_{\mathrm{a}}$ excitations, but they tend to overestimate the ${ }^{1} \mathrm{~L}_{\mathrm{b}}$ ones. ${ }^{35 / 122}$ In 2011, it was shown for the first time that global TD-DHDFAs partially solved this problem compared to CC2 reference data. ${ }^{35}$ In 2019 , we confirmed this finding once again when we analyzed the same 5 linear systems (from naphthalene to hexacene) with our

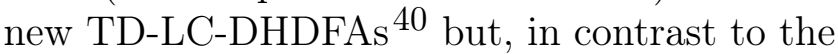
2011 work, we relied on high-level CR-EOM$\operatorname{CCSD}(\mathrm{T})^{123}$ data as well as the experimental reference values taken from Ref. 118. We found that $\omega \mathrm{B} 2 \mathrm{GP}-\mathrm{PLYP}$ was the best TDDFT method at that time with results that were slightly better than CC2. ${ }^{40}$ Herein, we re-analyze the same five systems with the same high-level reference values. All calculations 
Table 6: Statistical results (in eV) for the ${ }^{1} \mathrm{~L}_{a}-{ }^{1} \mathrm{~L}_{b}$ energy gap over the five linear polyacenes from naphthalene to hexacene using TDA (left) and full TD algorithms (right). ${ }^{\mathrm{a}}$

\begin{tabular}{|c|c|c|c|c|c|c|c|c|c|}
\hline \multirow[b]{2}{*}{ Method } & \multicolumn{4}{|c|}{ TDA-DHDFA } & \multirow[b]{2}{*}{ Method } & \multicolumn{4}{|c|}{ TD-DHDFA } \\
\hline & MD & MAD & RMSD & $\Delta_{\text {err }}$ & & MD & MAD & RMSD & $\Delta_{\text {err }}$ \\
\hline SOS-RSX-QIDH $^{b}$ & -0.03 & 0.03 & 0.03 & 0.01 & SCS- $\omega$ B2GP-PLYP & -0.02 & 0.03 & 0.03 & 0.08 \\
\hline SOS- $\omega$ B2GP-PLYP & -0.08 & 0.08 & 0.08 & 0.05 & SOS- $\omega$ PBEPP 86 & 0.03 & 0.03 & 0.04 & 0.06 \\
\hline SOS- $\omega$ PBEPP $86^{b}$ & -0.08 & 0.08 & 0.08 & 0.02 & SOS- $\omega$ B2GP-PLYP ${ }^{b}$ & 0.02 & 0.03 & 0.04 & 0.09 \\
\hline SOS- $\omega$ B88PP86 & -0.10 & 0.10 & 0.10 & 0.04 & SOS- $\omega$ B 88 PP 86 & -0.04 & 0.04 & 0.04 & 0.06 \\
\hline SCS- $\omega$ B2GP-PLYP & -0.11 & 0.11 & 0.11 & 0.03 & SCS- $\omega$ PBEPP 86 & -0.07 & 0.07 & 0.07 & 0.05 \\
\hline $\mathrm{SCS} / \mathrm{SOS}-\omega \mathrm{B} 2 \mathrm{PLYP}$ & -0.12 & 0.12 & 0.13 & 0.07 & SOS-PBE-QIDH & -0.08 & 0.08 & 0.08 & 0.02 \\
\hline SOS-PBE-QIDH & -0.13 & 0.13 & 0.13 & 0.02 & SCS- $\omega$ B 88 PP $86^{b}$ & -0.09 & 0.09 & 0.09 & 0.05 \\
\hline SCS- $\omega$ B 88 PP 86 & -0.14 & 0.14 & 0.14 & 0.04 & $\mathrm{SCS} / \mathrm{SOS}-\omega \mathrm{B} 2 \mathrm{PLY} \mathrm{P}^{\mathrm{b}}$ & -0.09 & 0.09 & 0.10 & 0.09 \\
\hline SOS-B2GP-PLYP21 & -0.17 & 0.17 & 0.17 & 0.04 & SCS-RSX-QIDH & -0.13 & 0.13 & 0.13 & 0.03 \\
\hline SCS- $\omega$ PBEPP 86 & -0.17 & 0.17 & 0.17 & 0.01 & SOS-RSX-QIDH & 0.14 & 0.14 & 0.14 & 0.09 \\
\hline SCS-PBE-QIDH & -0.18 & 0.18 & 0.18 & 0.02 & SCS-PBE-QIDH & -0.14 & 0.14 & 0.14 & 0.02 \\
\hline SCS-B2GP-PLYP21 & -0.18 & 0.18 & 0.19 & 0.04 & SOS-B2GP-PLYP21 & -0.19 & 0.19 & 0.19 & 0.02 \\
\hline RS-B88-LYP & -0.23 & 0.23 & 0.24 & 0.19 & SCS-B2GP-PLYP21 & -0.21 & 0.21 & 0.21 & 0.01 \\
\hline RS-PBE-PBE & -0.23 & 0.23 & 0.24 & 0.20 & $\omega$ PBEPP 86 & -0.23 & 0.23 & 0.23 & 0.05 \\
\hline RS-PBE-P86 & -0.23 & 0.23 & 0.24 & 0.20 & $\omega \mathrm{B} 88 \mathrm{PP} 86$ & -0.26 & 0.26 & 0.26 & 0.03 \\
\hline SCS-RSX-QIDH & -0.25 & 0.25 & 0.25 & 0.05 & $\omega \mathrm{B} 2 \mathrm{GP}-\mathrm{PLYP}$ & -0.27 & 0.27 & 0.27 & 0.02 \\
\hline$\omega$ РВЕРP 86 & -0.26 & 0.26 & 0.26 & 0.05 & RSX-QIDH & -0.31 & 0.31 & 0.31 & 0.03 \\
\hline$\omega \mathrm{B} 88 \mathrm{PP} 86$ & -0.27 & 0.27 & 0.27 & 0.03 & $\omega \mathrm{B} 2 \mathrm{PLYP}$ & -0.32 & 0.32 & 0.32 & 0.04 \\
\hline$\omega \mathrm{B} 2 \mathrm{GP}-\mathrm{PLYP}$ & -0.28 & 0.28 & 0.28 & 0.01 & B2GP-PLYP & -0.35 & 0.35 & 0.35 & 0.05 \\
\hline$\omega \mathrm{B} 2 \mathrm{PLYP}$ & -0.28 & 0.28 & 0.28 & 0.05 & PBE-QIDH & -0.38 & 0.38 & 0.38 & 0.04 \\
\hline SCS/SOS-B2PLYP21 & -0.29 & 0.29 & 0.29 & 0.07 & SCS/SOS-B2PLYP21 & -0.43 & 0.43 & 0.43 & 0.01 \\
\hline RSX-QIDH & -0.31 & 0.31 & 0.31 & 0.02 & B2PLYP & -0.44 & 0.44 & 0.44 & 0.03 \\
\hline B2GP-PLYP & -0.33 & 0.33 & 0.33 & 0.04 & RS-B88-LYP ${ }^{\mathrm{C}}$ & - & - & - & - \\
\hline PBE-QIDH & -0.36 & 0.36 & 0.36 & 0.03 & RS-PBE-PBE ${ }^{c}$ & - & - & - & - \\
\hline B2PLYP & -0.36 & 0.36 & 0.36 & 0.03 & RS-PBE-P $86^{\mathrm{C}}$ & - & - & - & - \\
\hline
\end{tabular}

a All calculations were performed with the cc-pVTZ AO basis set.The values for the RS-DH methods were taken from Ref. 80, ${ }^{\mathrm{b}}$ These methods perform well for each one of the ${ }^{1} \mathrm{~L}_{\mathrm{a}}$ and ${ }^{1} \mathrm{~L}_{\mathrm{b}}$ states, as well as for the energetic splitting. ${ }^{c}$ TD-DHDFA data not available.

were performed with the cc-pVTZ AO basis set. Due to the lack of high-level reference data for triplet excitations in this test set, we present results not only using the TDA-DHDFA scheme but also for the full TD-DHDFA scheme, as no triplet-instability problems are expected. This allows us also to test the transferability of our SCS/SOS parameters to the full TD-DHDF scheme.

Regarding the energy splitting between the two states we see an improvement when SCS/SOS is applied, however, there are two potential reasons for this observation: (a) either both the ${ }^{1} \mathrm{~L}_{\mathrm{a}}$ and ${ }^{1} \mathrm{~L}_{\mathrm{b}}$ excitations improve and, therefore, the energy gap between them also agrees better with the reference, or (b) the excitations to both states are shifted similarly but their energy difference matches with the reference. Ideally, the situation described in (a) is more desirable than (b), however, the energy differences also provide information that is as important and valuable as the values for the individual states. Those methods that belong to the situation described in (a) are highlighted in footnote $b$ in Table 6, where we present the statistics for the energy gap over the five systems. The complete raw data and the statistics for each of the ${ }^{1} \mathrm{~L}_{\mathrm{a}}$ and ${ }^{1} \mathrm{~L}_{\mathrm{b}}$ states can be found in the SI.
Similar to previous test sets, most of our SCS/SOS variants occupy the best positions in the ranking, with the exception of SCSRSX-QIDH and SCS/SOS-B2PLYP21. For the TDA-DHDFA scheme, we identify one and three methods that fulfill the first and second definition of "chemical accuracy", respectively, namely SOS-RSX-QIDH $(\mathrm{RMSD}=0.03 \mathrm{eV})$, SOS- $\omega$ B2GP-PLYP (RMSD $=0.08 \mathrm{eV}$ ), SOS$\omega$ PBEPP86 (RMSD $=0.08 \mathrm{eV})$, and SOS$\omega \mathrm{B} 88 \mathrm{PP} 86(\mathrm{RMSD}=0.10 \mathrm{eV})$. Amongst those four methods, which all display outstanding results, we highlight SOS-RSX-QIDH and SOS$\omega \mathrm{PBEPP} 86$ as functionals that satisfy the situation described in (a), i.e., the simultaneous improvement of both types of excitations. Eight other methods follow with RMSDs below $0.2 \mathrm{eV}$, the best of which are SCS- $\omega$ B2GPPLYP, SCS/SOS- $\omega$ B2PLYP, SOS-PBE-QIDH, SCS- $\omega$ B88PP86. The three RS-DH methods share an RMSD of $0.24 \mathrm{eV}$, followed by SCSRSX-QIDH with a value of $0.25 \mathrm{eV}$. Next, we find the unscaled methods $\omega$ PBEPP 86 , $\omega B 88$ PP86 , $\omega$ B2GP-PLYP, $\omega$ B2PLYP, and SCS/SCS-B2PLYP21, all of which are the remaining methods with RMSDs below $0.3 \mathrm{eV}$.

For the TD-DHDFA scheme, the picture is not too different, since most of the SCS/SOS methods are at the top of the ranking, with 
SCS/SOS-B2PLYP21 being the only exception. It is noteworthy that we now see eight methods that fulfill the second definition of "chemical accuracy", of which the best-four methods also fulfill the first definition (see Table 6); these are: SCS- $\omega$ B2GP-PLYP, SOS- $\omega$ PBEPP86, SOS- $\omega$ B2GP-PLYP, and SOS- $\omega$ B88PP86, SCS- $\omega$ PBEPP86 , SOS-PBE-QIDH, SCS$\omega \mathrm{B} 88 \mathrm{PP} 86$, and finally SCS/SOS- $\omega$ B2PLYP. Among those methods, SOS- $\omega$ B2GP-PLYP, SCS- $\omega$ B88PP86, and SCS/SOS- $\omega$ B2PLYP belong to category (a). Interestingly, SCS-RSXQIDH follows this time with a good RMSD of $0.13 \mathrm{eV}$, closely followed by its SOS variant and SCS-PBE-QIDH. SOS-B2GP-PLYP21 is the last method with an RMSD below 0.2 eV. Similarly to the TDA-DHDFA analysis,

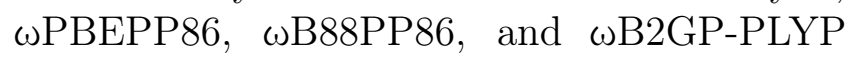
display RMSDs above $0.2 \mathrm{eV}$. The remaining unscaled methods and SCS/SOS-B2PLYP21 yield RMSDs above $0.3 \mathrm{eV}$.

Regarding the correct energetic order of the states, only a few methods give the wrong order for anthracene with respect to the experimental data, where the ${ }^{1} \mathrm{~L}_{\mathrm{a}}-{ }^{1} \mathrm{~L}_{\mathrm{b}}$ experimental gap is $-0.04 \mathrm{eV}$. For the TDA-DHDFA scheme, only three methods fail, namely, SOS$\omega B 2$ GP-PLYP (gap of $0.01 \mathrm{eV}$ ), SOS-RSXQIDH (0.06 eV), and SOS- $\omega$ PBEPP86 (0.01 $\mathrm{eV})$. For the TD-DHDFA scheme, seven methods fail in this regard: SCS- and SOS- $\omega$ B2GPPLYP (0.06 and $0.10 \mathrm{eV}$, respectively), SOSPBE-QIDH (0.01 eV), SOS-RSX-QIDH (0.21 $\mathrm{eV})$, SOS- $\omega$ B88PP86 (0.04 eV), SCS- and SOS$\omega$ PBEPP86 (0.01 and $0.11 \mathrm{eV}$, respectively). Note that the CR-EOM-CCSD(T) method also yields the wrong order of states for anthracene $(0.10 \mathrm{eV})$ compared to experimental data.

In summary, to the best of our knowledge the results for some of the new methods developed herein constitute the best $\mathrm{TD}(\mathrm{A})$-DFT results published to date for these systems.

\subsection{An Update on Charge- Transfer Excitations}

The LC methods were mainly developed with the aim to solve the charge-transfer (CT) problem of TD-DFT, and we proceed with a full assessment of our "updated" CT set presented in Ref. 40, In 2020, we modified this set and here we use the same systems again. Those are: 2,4-dichloro-6-p-N,N-diethylaminobiphenylyl1,3,5-triazine (DBQ), 34 para-nitroaniline (pNA), $\stackrel{124}{ }$ dimethylaminobenonitrile (DMABN), 124 benzene-tetracyanoethylene complex (BTCNE), 124 hydrogen chloride $(\mathrm{HCl}), 113$ a dipeptide, 125 a $\beta$-dipeptide, 125 and finally N-phenylpyrrole (PP). ${ }^{126}$ Similar to the previous set, we also present full TD-DHDFA results due to the lack of data for singlettriplet excitations. The high-level reference data used in this set are: theoretically backcorrected from the experiment (DBQ), linearresponse CCSDR(3) (pNA and peptides), EOM-CCSD(T) (DMABN and B-TCNE), and linear-response $\mathrm{CC} 3(\mathrm{HCl})$, respectively (also see SI).

When inspecting Table 7, it should not come as a surprise that global TD(A)-DHDFAs are not suitable for describing CT excitations. We point out that the opposite has been claimed in $2020^{73}$ but disproven by us on three occasions. 40 41 43 This study constitutes the fourth occasion. As already discussed by us for arylTCNE complexes, 43 we observe again that global DHDFAs artificially benefit from the TDA-DHDFA scheme thanks to a systematic blue shift in the excitation energies. In addition, we see that the SCS or SOS variants of global methods reduce the RMSD by half, e.g, TD(A)-B2PLYP and TD(A)-B2GP-PLYP.

Inspecting the TDA-DHDFA results for LC-DHDFAs, SOS-RSX-QIDH is the best tested method with an RMSD of $0.20 \mathrm{eV}$, closely followed by SOS- $\omega$ B88PP86 and SOS-

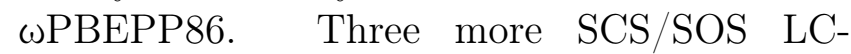
DHDFAs follow before the first global DHDFA appears in the ranking, namely, SCS-B2GPPLYP21 with an RMSD of $0.26 \mathrm{eV}$, sharing the same value as SCS/SOS- $\omega$ B2PLYP. Next, we have SCS- $\omega$ PBEPP86, SOS-B2GP-PLYP21, PBE-QIDH's SCS and SOS variants, alongside SCS-RSX-QIDH with values ranging from 0.27 to $0.29 \mathrm{eV}$. Most of the remaining methods display RMSDs that range from $0.30 \mathrm{eV}$ (PBEQIDH, SCS-PBE-QIDH, and $\omega B 88$ PP86) to $0.66 \mathrm{eV}$ (B2PLYP). 
Table 7: Statistical results (in eV) for the full CT set using TDA (left) and full TD algorithms (right). ${ }^{\text {a }}$

\begin{tabular}{|c|c|c|c|c|c|c|c|c|c|}
\hline \multirow[b]{2}{*}{ Method } & \multicolumn{4}{|c|}{ TDA-DHDFA } & \multirow[b]{2}{*}{ Method } & \multicolumn{4}{|c|}{ TD-DHDFA } \\
\hline & MD & MAD & RMSD & $\Delta_{\text {err }}$ & & MD & MAD & RMSD & $\Delta_{\text {err }}$ \\
\hline SOS-RSX-QIDH & 0.05 & 0.17 & 0.20 & 0.70 & SOS- $\omega$ B2GP-PLYP & 0.03 & 0.12 & 0.15 & 0.47 \\
\hline SOS- $\omega$ B88PP 86 & 0.05 & 0.15 & 0.21 & 0.80 & SCS- $\omega$ B2GP-PLYP & 0.00 & 0.12 & 0.16 & 0.57 \\
\hline SOS- $\omega$ PBEPP 86 & 0.01 & 0.16 & 0.22 & 0.82 & SCS/SOS- $\omega$ B2PLYP & 0.07 & 0.12 & 0.16 & 0.48 \\
\hline SCS- $\omega$ B2GP-PLYP & 0.11 & 0.17 & 0.23 & 0.75 & SOS- $\omega$ B 88 PP 86 & -0.04 & 0.14 & 0.18 & 0.68 \\
\hline SOS- $\omega$ B2GP-PLYP & 0.14 & 0.18 & 0.23 & 0.65 & SCS- $\omega \mathrm{B} 88 \mathrm{PP} 86$ & 0.00 & 0.13 & 0.19 & 0.73 \\
\hline SCS- $\omega \mathrm{B} 88 \mathrm{PP} 86$ & 0.09 & 0.18 & 0.24 & 0.85 & SOS-RSX-QIDH & -0.06 & 0.16 & 0.19 & 0.53 \\
\hline SCS-B2GP-PLYP21 & -0.08 & 0.19 & 0.26 & 0.94 & SOS- $\omega$ PBEPP 86 & -0.08 & 0.16 & 0.20 & 0.69 \\
\hline $\mathrm{SCS} / \mathrm{SOS}-\omega \mathrm{B} 2 \mathrm{PLYP}$ & 0.18 & 0.21 & 0.26 & 0.66 & $\omega \mathrm{B} 2 \mathrm{PLYP}$ & 0.15 & 0.18 & 0.23 & 0.61 \\
\hline SCS- $\omega$ PBEPP 86 & -0.03 & 0.21 & 0.27 & 1.02 & $\omega \mathrm{B} 2 \mathrm{GP}-\mathrm{PLYP}$ & 0.15 & 0.19 & 0.23 & 0.64 \\
\hline SOS-B2GP-PLYP21 & -0.13 & 0.21 & 0.28 & 0.95 & $\omega \mathrm{B} 88 \mathrm{PP} 86$ & 0.01 & 0.17 & 0.24 & 0.92 \\
\hline SOS-PBE-QIDH & -0.15 & 0.22 & 0.29 & 0.89 & SCS-RSX-QIDH & -0.03 & 0.18 & 0.24 & 0.87 \\
\hline SCS-RSX-QIDH & 0.07 & 0.22 & 0.29 & 1.06 & SCS-B2GP-PLYP21 & -0.15 & 0.20 & 0.25 & 0.78 \\
\hline SCS-PBE-QIDH & -0.15 & 0.23 & 0.30 & 0.95 & $\omega \mathrm{PBEPP} 86$ & 0.00 & 0.19 & 0.26 & 0.96 \\
\hline PBE-QIDH & -0.05 & 0.24 & 0.30 & 1.07 & SCS- $\omega$ PBEPP 86 & -0.12 & 0.21 & 0.27 & 0.89 \\
\hline$\omega \mathrm{B} 88 \mathrm{PP} 86$ & 0.09 & 0.24 & 0.30 & 1.04 & SOS-B2GP-PLYP21 & -0.20 & 0.23 & 0.29 & 0.79 \\
\hline$\omega \mathrm{PBEPP} 86$ & 0.08 & 0.25 & 0.31 & 1.09 & PBE-QIDH & -0.08 & 0.23 & 0.29 & 0.92 \\
\hline SCS/SOS-B2PLYP21 & -0.12 & 0.26 & 0.34 & 1.14 & SOS-PBE-QIDH & -0.18 & 0.23 & 0.30 & 0.81 \\
\hline$\omega \mathrm{B} 2 \mathrm{GP}-\mathrm{PLYP}$ & 0.26 & 0.30 & 0.34 & 0.84 & SCS-PBE-QIDH & -0.17 & 0.24 & 0.31 & 0.84 \\
\hline$\omega \mathrm{B} 2 \mathrm{PLYP}$ & 0.27 & 0.29 & 0.35 & 0.80 & RSX-QIDH & 0.28 & 0.29 & 0.33 & 0.59 \\
\hline B2GP-PLYP & -0.28 & 0.34 & 0.44 & 1.17 & SCS/SOS-B2PLYP21 & -0.20 & 0.27 & 0.34 & 0.98 \\
\hline RSX-QIDH & 0.40 & 0.41 & 0.45 & 0.80 & B2GP-PLYP & -0.34 & 0.36 & 0.45 & 1.02 \\
\hline B2PLYP & -0.53 & 0.57 & 0.66 & 1.33 & B2PLYP & -0.58 & 0.59 & 0.69 & 1.18 \\
\hline
\end{tabular}

a The "dipeptide", " $\beta$-peptide", and PP calculations were performed with the cc-pVTZ AO basis set. The B-TCNE, DMABN, pNA, and $\mathrm{HCl}$ calculations were performed with the aug-cc-pVTZ AO basis set. The DBQ calculation was performed with the def2-TZVPP 127 AO basis set.

For the TD-DHDFA results, it is noticeable that $\omega B 2$ GP-PLYP's SCS and SOS variants have significantly lowered their RMSDs compared to the TDA-DHDFA values, namely from 0.23 to $0.15 / 0.16 \mathrm{eV}$, which position them as the best TD-DHDFA methods. In total, there are seven new SCS/SOS-LC-DHDFA methods that all have RMSDs below $0.2 \mathrm{eV}$ and surpass the previously reported best results for $\omega$ B2PLYP and $\omega$ B2GP-PLYP $(\mathrm{RMSD}=0.23 \mathrm{eV})$. The remaining methods in this ranking are listed in Table 7) similar to the TDA-DHDFA scheme, TD-B2PLYP is the worst DHDFA with an RMSD of $0.69 \mathrm{eV}$.

In Ref. 80, the RS-DH methods were assessed on a slightly modified version of our CT set ${ }^{40}$ and only for the TDA-DHDFA scheme. Therein, our peptides were excluded from the analysis. In order to compare our methods with those, we included an additional analysis that omits those systems in the SI. We briefly mention that there is a small decrease in the RMSDs for some methods, for instance, a reduction of $0.02 \mathrm{eV}$ for SOS-RSX-QIDH, $0.03 \mathrm{eV}$ for SOSwB88PP86. However, the general trend remains the same. The RS-DH methods are positioned after the SCS and SOS variants of our own LC-DHDFAs, with RMSDs slightly above $0.30 \mathrm{eV}$.

\section{Cross-Validation for Ver- tical Singlet-Triplet Exci- tations}

After having presented promising results for vertical singlet-singlet excitations, we shift our attention to the discussion of vertical singlettriplet excitations. The first study of such excitations was done in 2007 for the TDA-B2PLYP functional, $\frac{18}{18}$ where Grimme and Neese examined 22 excitations in seven different systems based on experimental data. In 2020, we assessed the TDA-DHDFAs idea for the second time and, for the first time, TD-DHDFAs for such excitations in a more comprehensive study. $\frac{41}{}$ Our study showed that like other functionals TD-DHDFAs are not useful for such excitations, but that TDA-DHDFAs can provide accurate results. Herein, we follow up on our study from 2020 and present the first study of such excitations with SCS and SOS techniques applied to TDA-DHDFAs. Note that there is no data available for such excitations for the new RS-DH methods and they will not be considered in the following.

\subsection{The Loos and Jacquemin Benchmark Set}

The original benchmark set by Loos, Jacquemin, and co-workers comprises 47 


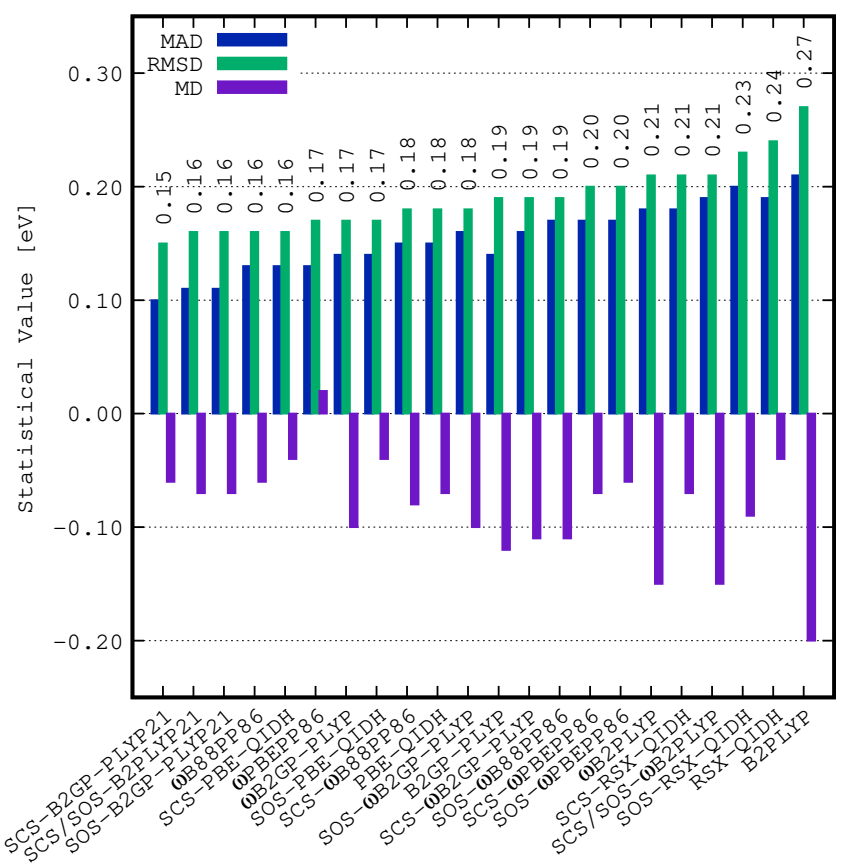

Figure 5: Statistical values (in eV) for the SO-TN transitions for the Loos and Jacquemin set, namely RMSDs (values shown over each bar), MADs, and MDs. The aug-cc-pVTZ AO basis set was used in all cases.

singlet-triplet transitions, of which we selected 35 transitions (23 local valence, 12 Rydberg, and one CT state) in 13 different molecules, which have not been included in other herein discussed sets. All references are of CC3/augcc-pVTZ quality and taken from Ref. 113, except for $\mathrm{HCl}$, which was calculated by us and reported in Ref. 41 .

\subsubsection{All Singlet-Triplet Excitations in this set (S0-TN)}

Fig. 5 shows the same trend as in the updated Gordon set in Section 4.2.3, thus, confirming the reliability of our new methods. Similar to the updated Gordon set, our methods again perform very well, with SCS-B2GP-PLYP21 being the first method in our ranking $(0.15 \mathrm{eV})$, closely followed by SCS/SOS-B2PLYP21, SOSB2GP-PLYP21, $\omega$ B88PP86, and SCS-PBEQIDH. Furthermore, $\omega$ PBEPP86, $\omega \mathrm{B} 2 \mathrm{GP}-$ PLYP, SOS-PBE-QIDH, SCS- $\omega$ B88PP86, the unscaled PBE-QIDH, and SOS- $\omega$ B2GP-PLYP perform very well with RMSDs of about 0.18 $\mathrm{eV}$. The last four methods with RMSDs below $0.2 \mathrm{eV}$ are B2GP-PLYP, SCS- $\omega$ B2GPPLYP, SOS- $\omega$ B88PP86, and SCS- $\omega$ PBEPP86.

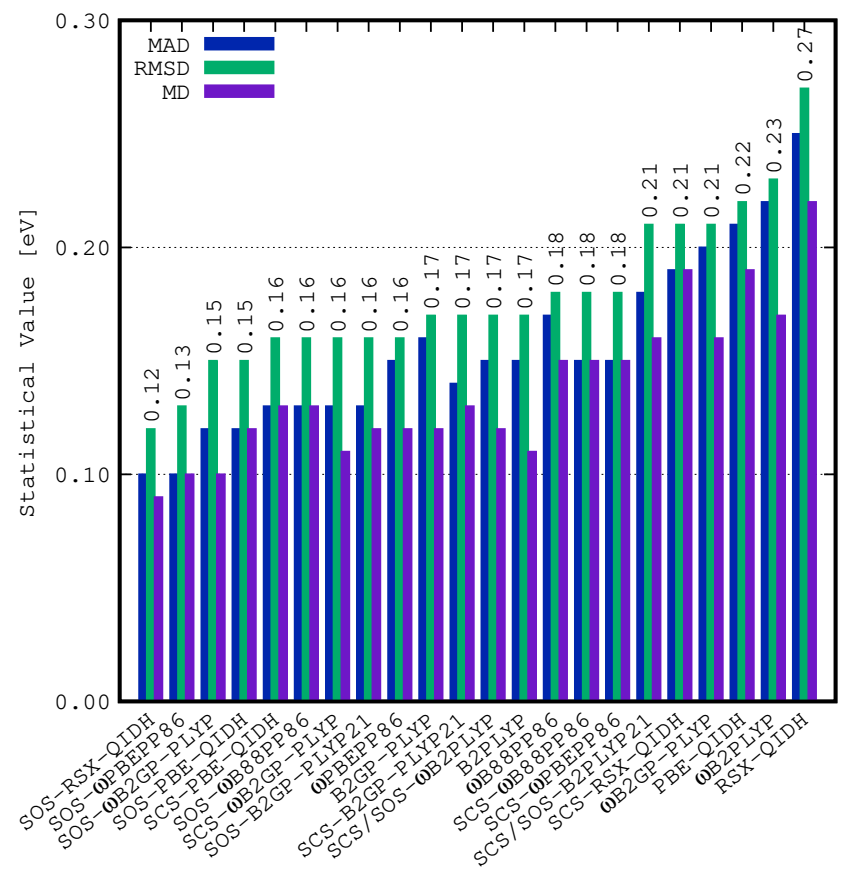

Figure 6: Statistical values (in eV) for the S1-T1 energy splitting on the Loos and Jacquemin set, namely RMSDs (values shown over each bar), MADs, and MDs. The aug-cc-pVTZ AO basis set was used in all cases.

Other functionals are shown in Fig. 5 and we just point out that we obtained the largest RMSDs for SOS-RSX-QIDH, its unscaled variant RSX-QIDH, and B2PLYP with values beyond $0.23 \mathrm{eV}$. The MDs display the same trend as in the updated Gordon set by showing a systematic underestimation for the S0-TN transitions.

\subsubsection{S1-T1 Analysis}

Despite global DHDFAs leading the S0-TN analysis, LC-DHDFAs are the best for the S1T1 energy splitting, as can be seen in Fig. 6. where SOS-RSX-QIDH and SOS- $\omega$ PBEPP86 display very good RMSDs of about $0.12 \mathrm{eV}$, slightly above the "chemical accuracy" threshold. Besides those two, there are other 14 methods that perform very well with RMSDs below $0.2 \mathrm{eV}$, six of which are SCS/SOS-LCDHDFAs (see Fig. 6). Surprisingly, there are two SCS/SOS methods that are positioned above $0.2 \mathrm{eV}$, namely, SCS/SOS-B2PLYP21 which was the second-best in the S0-TN analysis, and SCS-RSX-QIDH. The worst performer is the unscaled RSX-QIDH with an RMSD of $0.27 \mathrm{eV}$. 


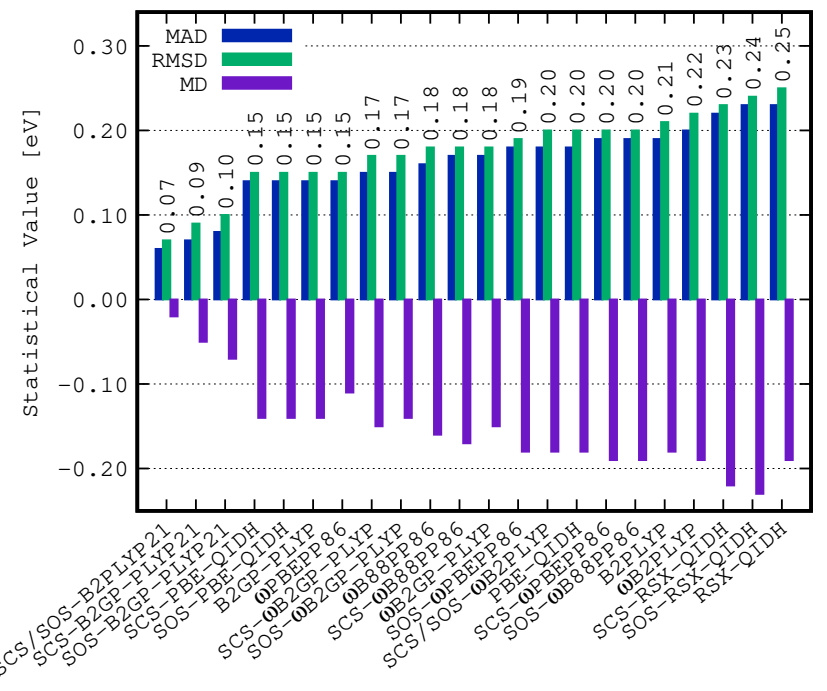

Figure 7: Statistical values (in eV) for the S0-TN transitions for the "Exotic-Molecules" set, namely RMSDs (values shown over each bar), MADs, and MDs. The aug-cc-pVTZ AO basis set was used in all cases.

The MDs follow the same trend as in the updated Gordon set, with a systematic underestimation in the excitation energies for the S0-TN analysis, whereas there is a systematic overestimation for the S1-T1 energy splitting. In the remaining sections, we will see that this is a general trend and cease to comment on MDs.

\subsection{The "Exotic-Molecules" Set}

This set by Loos, Jacquemin, and co-workers 114 consists of 11 singlet-triplet transitions of localvalence character in seven different systems, all of high-level CC3/aug-cc-pVTZ quality.

\subsubsection{All Singlet-Triplet Excitations in this set (S0-TN)}

Similar to Section 6.1.1, the SCS and SOS variants of B2PLYP and B2GP-PLYP are also the best methods here, with low RMSDs that range from $0.07 \mathrm{eV}$ for SCS/SOS-B2PLYP21 to 0.10 eV for SOS-B2GP-PLYP21 (Fig. 7). Somewhat higher in energy, SCS- and SOS-PBE-QIDH, B2GP-PLYP, and $\omega$ PBEPP86 share the same RMSD of $0.15 \mathrm{eV}$. $\omega B 2$ GP-PLYP's SCS and SOS variants, $\omega \mathrm{B} 88 \mathrm{PP} 86$, SOS- $\omega \mathrm{B} 88 \mathrm{PP} 86$, and the unscaled $\omega B 2 G P-P L Y P$ follow next in the ranking. The last DHDFA with an RMSD below $0.2 \mathrm{eV}$ is SOS- $\omega$ PBEPP86 with an RMSD of $0.19 \mathrm{eV}$. The remaining scaled and unscaled

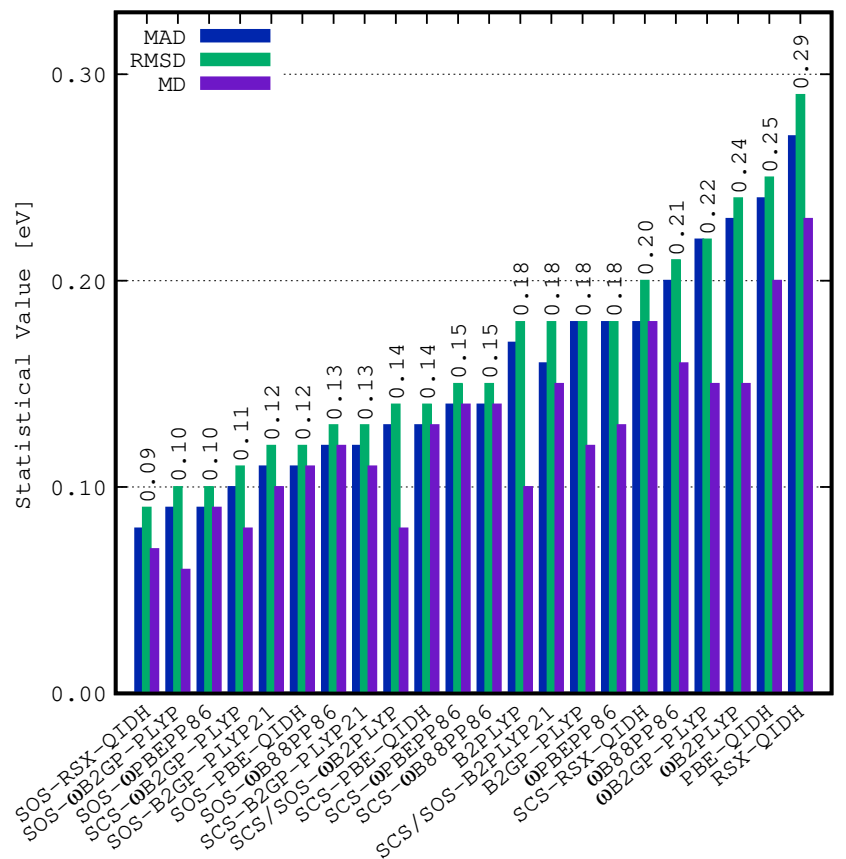

Figure 8: Statistical values (in eV) for the S1-T1 energy splitting on the "Exotic-Molecules" set, namely RMSDs (values shown over each bar), MADs, and MDs. The aug-cc-pVTZ AO basis set was used in all cases.

methods display results that range from 0.20 $\mathrm{eV}$ (SCS/SOS- $\omega \mathrm{B} 2 \mathrm{PLYP})$ to $0.25 \mathrm{eV}$ (RSXQIDH).

\subsubsection{S1-T1 Analysis}

The S1-T1 splitting shows the same behavior as in the previous set (Fig. 8). The SOS variants of RSX-QIDH, $\omega$ B2GP-PLYP, and $\omega \mathrm{PBEPP} 86$ are the best three performers with RMSDs within the chemical accuracy threshold of $0.10 \mathrm{eV}$. Other methods that also display excellent results are SCS- $\omega$ B2GP-PLYP, B2GP-PLYP, PBE-QIDH, and SOS- $\omega$ B88PP86 with values near $0.12 \mathrm{eV}$. SCS/SOS- $\omega$ B2PLYP, SCS-PBE-QIDH, SCS- $\omega$ PBEPP86, and SCS$\omega B 88$ PP86 perform similarly with RMSDs of up to $0.15 \mathrm{eV}$. B2PLYP is the first unscaled method in the list and shares the same RMSD of $0.18 \mathrm{eV}$ with SCS/SOS-B2PLYP21, B2GPPLYP, and $\omega P B E P P 86$. The remaining methods have RMSDs ranging from $0.20 \mathrm{eV}$ (SCSRSX-QIDH) to $0.29 \mathrm{eV}$ (RSX-QIDH). 


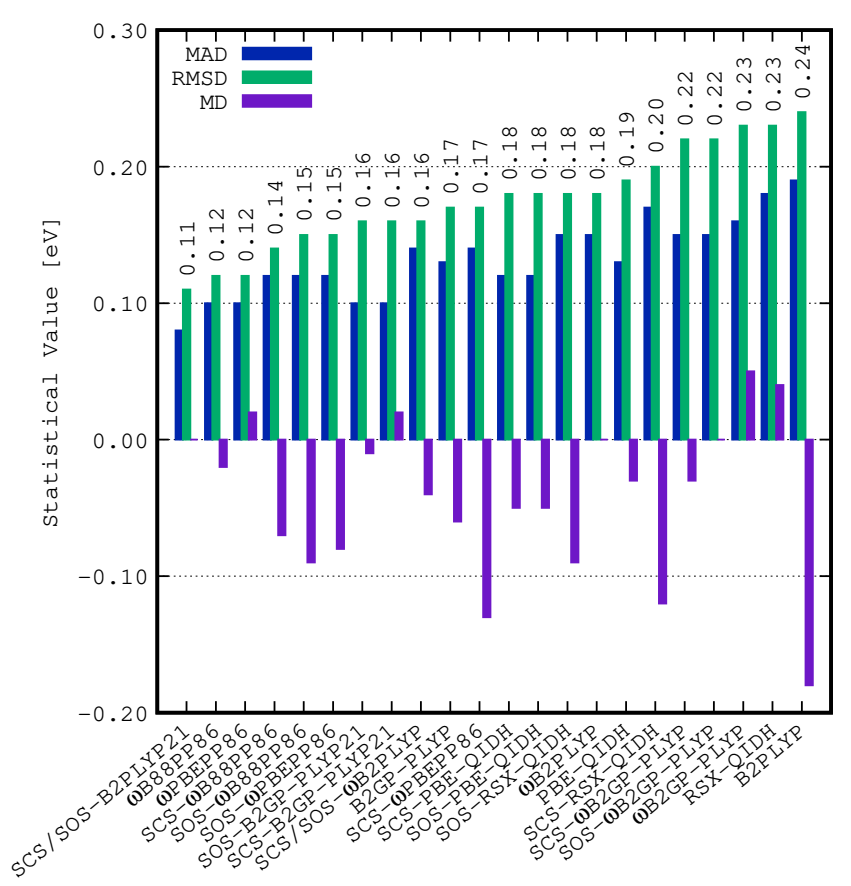

Figure 9: Statistical values (in eV) for the SO-TN transitions for the Thiel set, namely RMSDs (values shown over each bar), MADs, and MDs. The TZVP AO basis set was used in all cases.

\subsection{The Thiel Benchmark Set}

The original set by Thiel and co-workers comprises 63 triplet excitations with CC3/TZVP27 reference values. However, most of the molecules have already been addressed in our preceding analyses and therefore we decided to study only six molecules that have not been assessed before, namely, acetamide, benzoquinone, imidazole, naphthalene, norbornadiene, and octatetraene. For these six molecules, a total of 25 local-valence excitations need to be analyzed.

\subsubsection{All Singlet-Triplet Excitations in this set (S0-TN)}

The RMSDs are below $0.2 \mathrm{eV}$ for almost every tested method (see Fig. 9). SCS/SOSB2PLYP21 and the new unscaled DHDFAs, $\omega$ B88PP86 and $\omega$ PBEPP86, are positioned as the best three methods with RMSDs slightly above the chemical accuracy threshold. There are 13 methods with RMSDs below $0.2 \mathrm{eV}$, six of which are SCS/SOS-LC-DHDFAs. Surprisingly, we find wB2GP-PLYP's SCS and SOS variants among the worst-performing methods with an RMSD of $0.22 \mathrm{eV}$, followed by its un-

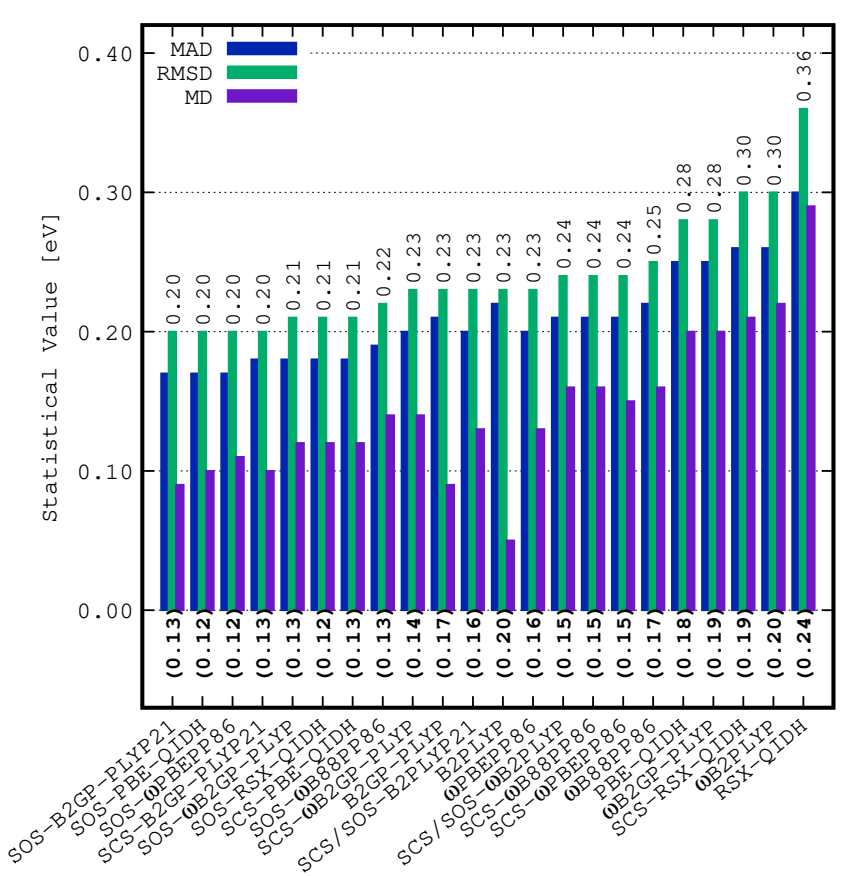

Figure 10: Statistical values (in eV) for the S1-T1 energy splitting on the Thiel set, namely RMSDs (values shown over each bar), MADs, and MDs. The TZVP AO basis set was used in all cases. The methods are sorted according to their overall RMSDs by considering the singlet excited state of $\pi-\pi^{*}$ type $\left(2{ }^{1} A^{\prime}\right.$ symmetry) in imidazole. RMSDs based on the $n-\pi^{*}$ transition in imidazole are shown in parentheses.

scaled parent $\omega$ B2GP-PLYP, RSX-QIDH, and B2PLYP (more details in Fig. 9).

\subsubsection{S1-T1 Analysis}

Note that Thiel and co-workers argued that the first singlet-singlet excited state in imidazole was of $\mathrm{n}-\pi^{*}$ type and has $1^{1} \mathrm{~A}$ " symmetry. 27 However, after closer inspection of their CC3 reference data provided in the same work we found that the first singlet excited state should be of $\pi-\pi^{*}$ character and have $2^{1} \mathrm{~A}^{\prime}$ symmetry. In light of this finding, Fig. 10 includes both analyses with the values based on the $n-\pi^{*}$ transition given in parentheses.

The RMSDs for the S1-T1 splitting are higher than in previous sets when the $\pi-\pi^{*}$ type singlet excited state for imidazole is taken into account: now the RMSDs range from 0.20 eV (SOS- and SCS-B2GP-PLYP21, SOS-PBEQIDH, and SOS- $\omega$ PBEPP86) to $0.36 \mathrm{eV}$ (RSXQIDH). Nevertheless, most of the SCS or SOS variants still display similar behavior as in previous sets, with SCS-RSX-QIDH being the only exception with an RMSD of $0.30 \mathrm{eV}$. Similarly, 
the unscaled PBE-QIDH $(0.28 \mathrm{eV}), \omega \mathrm{B} 2 \mathrm{GP}$ PLYP (0.28 eV), $\omega B 2 P L Y P(0.30 \mathrm{eV})$, and RSX-QIDH are positioned last.

If we now consider the $\mathrm{n}-\pi^{*}$ type singlet excited state in imidazole, the RMSDs (values in parentheses in Fig. 10 are greatly improved by about $0.08 \mathrm{eV}$. In this picture, the best six methods are all SOS variants with RMSDs of $0.12-0.13 \mathrm{eV}$. Next, we identify another six methods all of which are SCS variants with RMSDs of up to $0.15 \mathrm{eV}$. $\omega \mathrm{PBEPP} 86$ is the first unscaled DHDFA and shares the same RMSD as SCS/SOS-B2PLYP21 of $0.16 \mathrm{eV}$. The unscaled DHDFAs $\omega$ B88PP86, B2GP-PLYP, PBE-QIDH, $\omega B 2$ GP-PLYP, and SCS-RSXQIDH are the last five methods with RMSDs below $0.2 \mathrm{eV}$. Lastly, B2PLYP, $\omega B 2 P L Y P$, and RSX-QIDH are positioned last.

\section{Overall Performance}

In the spirit of the GMTKN55 database for ground state main-group thermochemistry, kinetics, and noncovalent interactions ${ }^{8}$ we perform two additional analyses that help identify those methods that distinguish themselves from others according to their overall performance and robustness. In order to achieve such an analysis, we opted for two different strategies: (a) we order the TDA-DHDFAs by their RMSDs for each set and then rank them according to how many times a certain method yields the three best or three worst RMSDs and (b) we rank them by their averaged MAD or RMSD $(\overline{\mathrm{MAD}} / \overline{\mathrm{RMSD}})$ in the TDA-DHDFA framework according to

$$
\overline{\mathrm{MAD}} / \overline{\mathrm{RMSD}}=\frac{\sum_{\mathrm{i}}^{16}(\mathrm{MAD} / \mathrm{RMSD})_{\mathrm{i}}}{16},
$$

where the sum runs over each of the 16 individual local-valence, Rydberg, and CT excitation subsets and (MAD/RMSD) $)_{i}$ is the $\mathrm{MAD} / \mathrm{RMSD}$ of the respective method for the $i^{\text {th }}$ set. For those sets for which we broke down previous analyses into overall, local-valence, and Rydberg excitations, we have included the statistics of the last two cases to avoid any bias

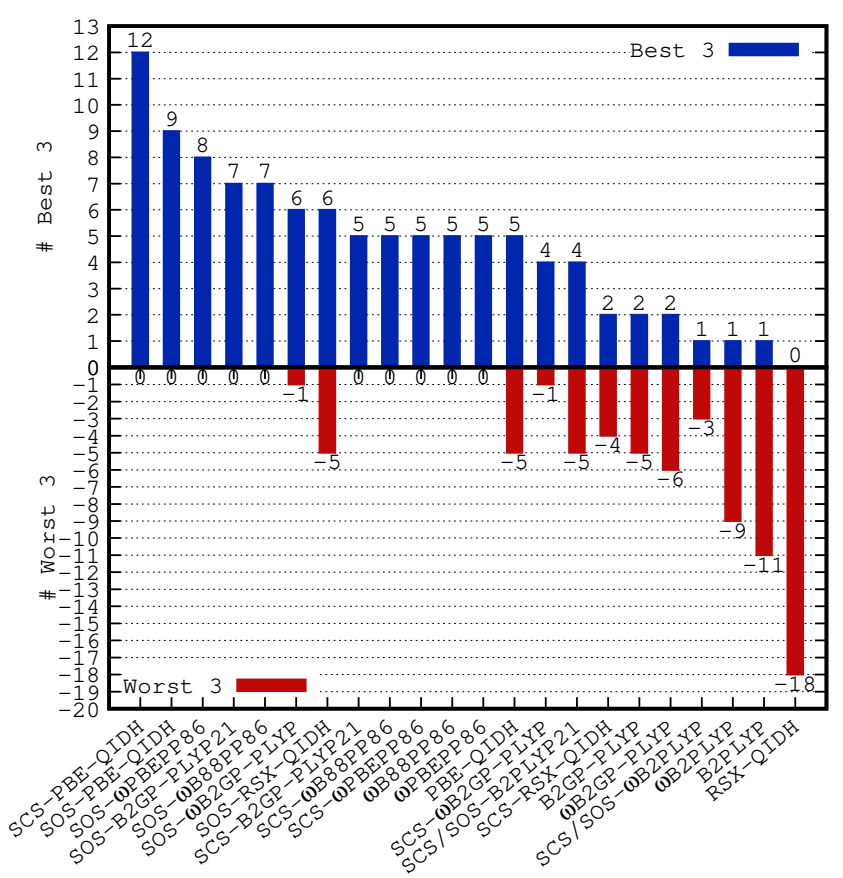

Figure 11: Analysis of how many times a method yields the three best and three worst RMSDs in a benchmark (sub)set.

due to possible double counting.

\subsection{Top-Three Best/Worst Anal- ysis}

We begin with some general comments on how this analysis was performed. In almost every set we observed that multiple methods yielded the same RMSD. For instance, in the localvalence analysis of the $\omega$ B2(GP-)PLYP training set, there are four methods that share the best RMSD of $0.10 \mathrm{eV}$ (SOS- $\omega$ B88PP86, SOSPBE-QIDH, and $\omega$ B2GP-PLYP's SCS and SOS variants). Therefore, we assign the first position to each of them. We repeat the same strategy also for the second, third, and last three positions for every set. The outcome of our analysis is shown in Fig. 11.

PBE-QIDH's SCS and SOS variants are some of the most robust functionals in this work, where SCS-PBE-QIDH is on the top twelve times and SOS-PBE-QIDH nine times, respectively. However, we have to emphasize that neither of these methods outperform TDA-LCDHDFAs for CT transitions, and the difference is even larger if we consider the TD-LC-DHDFA results (see Section 5.6 and SI). Our new SOS$\omega \mathrm{PBEPP} 86$ is another well-performing func- 
tional and is one of the top-three methods eight times. It also comes with the advantage of performing well regardless of the type of excitation, incl. CT ones. If the nature of the transition is unknown, SOS- $\omega$ PBEPP86 is therefore robust enough to give reliable results. SOS$\omega$ B88PP86 and SOS-B2GP-PLYP21 can also be recommended, keeping in mind that the first outperforms the latter for CT transitions. SOS- $\omega$ B2GP-PLYP and SOS-RSX-QIDH are the first two methods that are somewhat less robust given that they rank among the worst three methods once and five times, respectively. Some other methods that we recommend are SCS- $\omega$ B88PP86, SCS- $\omega$ PBEPP86, SCS-B2GPPLYP21, $\omega$ PBEPP86, and $\omega B 88 P P 86$. Even though they only rank in the top three five times, they never appear among the worst. From the unscaled PBE-QIDH onward, the methods display less robust behavior and can not be recommended for future applications to unexplored problems if a functional of general robustness is required.

\subsection{Averaged MAD/RMSD Anal- ysis}

In Fig. 12, we present the $\overline{\mathrm{MAD}}$ and $\overline{\mathrm{RMSD}}$ values for the present work. Their trends seem to be similar to the previous analysis, where SCS and SOS-PBE-QIDH are on top of the list with good results of just $0.16 \mathrm{eV}$ for $\overline{\mathrm{RMSD}}$, followed by our new methods SOS- $\omega$ B88PP86 $(0.17 \mathrm{eV})$, SCS- $\omega$ B88PP86 (0.18 eV), SOS$\omega$ PBEPP86 (0.18 eV), and SCS- $\omega$ PBEPP86 $(0.18 \mathrm{eV})$. Other methods that we also recommend are SOS-B2GP-PLYP21, SCS and SOS- $\omega$ B2GP-PLYP. Nevertheless, SOS-B2GPPLYP21 should be used with care due to its inability to describe CT transitions. On the other hand, $\omega$ PBEPP86 and $\omega$ B88PP86 still show good results despite being unscaled methods and are the last two with $\overline{\mathrm{RMSD}}$ values below $0.2 \mathrm{eV}$. The remaining unscaled methods display values above $0.2 \mathrm{eV}$, with RSX-QIDH and B2PLYP being the ones with the poorest results of 0.31 and $0.33 \mathrm{eV}$, respectively. Even though SCS and SOS-PBE-QIDH are at the top of the list, our new SCS/SOS-LC-DHDFA methods

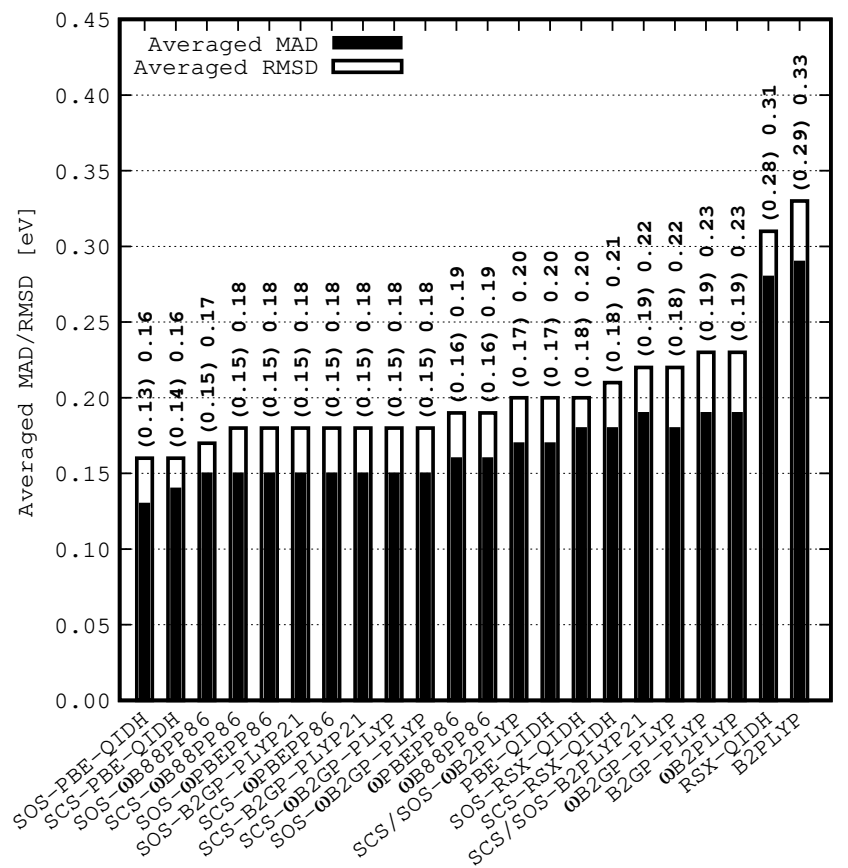

Figure 12: $\overline{\mathrm{MAD}} / \overline{\mathrm{RMSD}}$ analysis (in eV). The $\overline{\mathrm{RMSD}}$ s are shown as outlines and the MADs as solid bars. We also present their values above each bar, with the $\overline{M A D}$ in parentheses. The methods are sorted according to their $\overline{\mathrm{RMSD}}$.

have an increased $\overline{\mathrm{RMSD}}$ of only $0.01 \mathrm{eV}$ and they can provide more reliable results for systems that might have mixed valence-CT character (see Fig. 12). Indeed, our SCS/SOS-LCDHDFAs are more accurate not only for CT transitions but also for the PAHs.

\section{Conclusions}

This has been the second time that SCS and SOS variants have been introduced and assessed for double hybrid density functional approximations (DHDFAs) for the computation of electronic excitation energies that follow the initial definition by Grimme and Neese (see Eq.7). The main differences to the first work of this kind in 2017 39 are the incorporation of the latest LC-DHDFAs $40,80,97$ and the analysis of singlet-triplet excitations. We remind the reader that herein we already took into account the best TD(A)-DFT methods and improving them further poses a major challenge. We compared unscaled DHDFAs with new SCS and SOS variants and tested those on six benchmark sets that comprised 203 excitations in small to medium-sized molecules and different 
excitation types. While most of this work focused mostly on the Tamm-Dancoff approximation (TDA-DHDFAs), we also presented results based on full TD-DHDFAs for the PAH and CT benchmark sets.

We confirmed the good accuracy and robustness of DHDFAs for the computation of electronic excitation energies. In general, they showed the same accuracy as previous studies. The introduction of SCS and SOS caused a systematic red-shift in the excitation energies, contrary to the previous observations made by Schwabe and Goerigk in 2017. $\underline{39}$ Sometimes, the red-shift eliminated extreme outliers in the excitation energies, thus reducing error spans by up to $0.3 \mathrm{eV}$. The only exceptions to this are the BLYP-based global DHDFAs B2PLYP and B2GP-PLYP, which displayed a blue-shift in the excitation energies. One benefit of using SCS and SOS is a more balanced description compared to the reference values, with MDs usually being closer to zero. Our SCS and SOS variants also showed that it is not necessary to include a long-range correction in the perturbative part of a DHDFA to obtain reasonable results. In fact, the three methods following that approach were outperformed by many of our newly presented ones for singlet-singlet transitions (no data on those competing methods were available for triplet transitions).

One important finding is that we did not see any particular trend of when to prefer SCS over SOS or vice-versa. In fact, SCS and SOS variants present negligible differences in the RMSDs of up to $0.03 \mathrm{eV}$ when applied to DHDFAs. Note that this is contrary to some ground state wave function methods where SOS can lead to inferior results compared to SCS, compromising accuracy and efficiency. $\stackrel{710}{ }$ Accurate SOS-DHDFAs provide faster calculation of larger systems and might also open a route to more efficient methods for the optimization of excited-state geometries once the Laplace transform is applied, similar to its wave function counterpart.

While unscaled TD(A)-DHDFAs already constitute an improvement over conventional TD(A)-DFT methods, as conclusively shown in the literature, 18/28/33 36]40|41 we recommend specific new SCS- and SOS-based variants presented in this work for further testing and subsequent applications. Based on our averaged MAD/RMSD analysis, we highly recommend our SCS and SOS variants of PBEQIDH, $\omega$ B88PP86, and $\omega$ PBEPP86. That being said, SCS- and SOS-PBE-QIDH are not suitable for the computation of CT excitations, and the SCS/SOS- $\omega$ B88PP86 and SCS/SOS$\omega$ PBEPP86 should be employed instead. Indeed, the latter four methods can provide accurate excitation energies regardless of the type of transition, making them more robust for real applications to new systems. Finally, if only unscaled DHDFAs are available to the user, we strongly recommend $\omega \mathrm{B} 88 \mathrm{PP} 86$ as the bestunscaled method, which outperformed some other SCS and SOS methods presented in this work.

Our work showed once more that $\mathrm{TD}(\mathrm{A})$ DHDFAs have their place in the treatment of accurate electronic excitation energies. All our newly presented methods will be available in the upcoming release of ORCA5. Finally, we would like to encourage the implementation of TD(A)-DHDFAs with and without SCS/SOS into other standard software packages.

Acknowledgement M.C.-P. acknowledges a 'Melbourne Research Scholarship' by The University of Melbourne. L. G. acknowledges generous allocation of resources by the $\mathrm{Na}$ tional Computational Infrastructure (NCI) National Facility within the National Computational Merit Allocation Scheme (project ID: fk5) and Research Platform Services (ResPlat) at The University of Melbourne (Project No. punim0094). This research was also supported by the sustaining and strengthening merit-based access to the NCI LIEF Grant (LE190100021) facilitated by The University of Melbourne.

\section{Supporting Information Avail- able}

The Supplementary Information includes all references values, excitation energies and sta- 
tistical values for all tested cases and density functional approximations in two seperate xlsx files. Those files are available free of charge.

\section{References}

(1) Hohenberg, P.; Kohn, W. Inhomogeneous electron gas. Phys. Rev. B 1964, 136, 864-871.

(2) Kohn, W.; Sham, L. J. Self-consistent equations including exchange and correlation effects. Phys. Rev. 1965, 140, A1133-A1138.

(3) Runge, E.; Gross, E. K. U. Densityfunctional theory for time-dependent systems. Phys. Rev. Lett. 1984, 52, 9971000 .

(4) Bauernschmitt, R.; Ahlrichs, R. Treatment of electronic excitations within the adiabatic approximation of time dependent density functional theory. Chem. Phys. Lett 1996, 256, 454-464.

(5) Grimme, S. Semiempirical hybrid density functional with perturbative secondorder correlation. J. Chem. Phys. 2006, 124, 034108.

(6) Goerigk, L.; Grimme, S. Efficient and accurate double-hybrid-meta-GGA density functionals-evaluation with the extended GMTKN30 database for general main group thermochemistry, kinetics, and noncovalent interactions. J. Chem. Theory Comput. 2011, 7, 291-309.

(7) Goerigk, L.; Grimme, S. A thorough benchmark of density functional methods for general main group thermochemistry, kinetics, and noncovalent interactions. Phys. Chem. Chem. Phys. 2011, 13, 6670-6688.

(8) Goerigk, L.; Hansen, A.; Bauer, C.; Ehrlich, S.; Najibi, A.; Grimme, S. A look at the density functional theory zoo with the advanced GMTKN55 database for general main group thermochemistry, kinetics and noncovalent interactions. Phys. Chem. Chem. Phys. 2017, 19, 32184-32215.

(9) Mardirossian, N.; Head-Gordon, M. Survival of the most transferable at the top of jacob's ladder: defining and testing the $\omega \mathrm{B} 97 \mathrm{M}(2)$ double hybrid density functional. J. Chem. Phys. 2018, 148, 241736 .

(10) Mehta, N.; Casanova-Páez, M.; Goerigk, L. Semi-empirical or non-empirical double-hybrid density functionals: which are more robust? Phys. Chem. Chem. Phys. 2018, 20, 23175-23194.

(11) Kozuch, S.; Gruzman, D.; Martin, J. M. L. DSD-BLYP: A general purpose double hybrid density functional including spin component scaling and dispersion correction. J. Phys. Chem. C 2010, 114, 20801-20808.

(12) Kozuch, S.; Martin, J. M. L. DSDPBEP86: in search of the best doublehybrid DFT with spin-component scaled MP2 and dispersion corrections. Phys. Chem. Chem. Phys. 2011, 13, 2010420107.

(13) Kozuch, S.; Martin, J. M. L. Spincomponent-scaled double hybrids: an extensive search for the best fifth-rung functionals blending DFT and perturbation theory. J. Comput. Chem. 2013, 34, $2327-2344$.

(14) Santra, G.; Sylvetsky, N.; Martin, J. M. L. Minimally empirical doublehybrid functionals trained against the GMTKN55 database: revDSD-PBEP86$\mathrm{d} 4$, revDOD-PBE-d4, and DOD-SCANd4. J. Phys. Chem. C 2019, 123, 5129 5143 .

(15) Martin, J. M. L.; Santra, G. Empirical double-hybrid density functional theory: A 'third way' in between WFT and DFT. Isr. J. Chem. 2019, 60, 787-804. 
(16) Santra, G.; Cho, M.; Martin, J. M. L. Exploring avenues beyond revised DSD functionals: I. range separation, with xDSD as a special case. J. Phys. Chem. A 2021, published online, DOI: 10.1021/acs.jpca.1c01294 (accessed on 30 May 2021).

(17) Santra, G.; Semidalas, E.; Martin, J. M. L. Exploring avenues beyond revised DSD functionals: II. random-phase approximation and scaled MP3 corrections. J. Phys. Chem. A 2021, published online, DOI: 10.1021/acs.jpca.1c01294 (accessed on 30 May 2021).

(18) Grimme, S.; Neese, F. Double-hybrid density functional theory for excited electronic states of molecules. J. Chem. Phys. 2007, 12\%, 154116.

(19) Head-Gordon, M.; Rico, R. J.; Oumi, M.; Lee, T. J. A doubles correction to electronic excited states from configuration interaction in the space of single substitutions. Chemical Physics Letters 1994, 219, 21-29.

(20) Hirata, S.; Head-Gordon, M. Timedependent density functional theory within the Tamm-Dancoff approximation. Chem. Phys. Lett. 1999, 314, 291299.

(21) Benighaus, T.; DiStasio, R. A.; Lochan, R. C.; Chai, J.-D.; HeadGordon, M. Semiempirical double-hybrid density functional with improved description of long-range correlation. $J$. Phys. Chem. A 2008, 112, 2702-2712.

(22) Zhang, I. Y.; Xu, X. Reaching a uniform accuracy for complex molecular systems: long-range-corrected XYG3 doubly hybrid density functional. J. Phys. Chem. Lett. 2013, 4, 1669-1675.

(23) Cornaton, Y.; Fromager, E. Double hybrid density-functional theory using the coulomb-attenuating method. Int. J. Quantum Chem. 2014, 114, 1199-1211.
(24) Hedegård, E. D.; Heiden, F.; Knecht, S.; Fromager, E.; Jensen, H. J. A. Assessment of charge-transfer excitations with time-dependent, range-separated density functional theory based on longrange MP2 and multiconfigurational selfconsistent field wave functions. J. Chem. Phys. 2013, 139, 184308.

(25) Kalai, C.; Toulouse, J. A general range-separated double-hybrid densityfunctional theory. J. Chem. Phys. 2018, 148,164105 .

(26) Karton, A.; Tarnopolsky, A.; Lamère, J.F.; Schatz, G. C.; Martin, J. M. L. Highly accurate first-principles benchmark data sets for the parametrization and validation of density functional and other approximate methods. derivation of a robust, generally applicable, double-hybrid functional for thermochemistry and thermochemical kinetics. J. Phys. Chem. A 2008, 112, 12868-12886.

(27) Schreiber, M.; Silva-Junior, M. R.; Sauer, S. P. A.; Thiel, W. Benchmarks for electronically excited states: CASPT2, CC2, CCSD, and CC3. J. Chem. Phys. 2008, 128, 134110.

(28) Goerigk, L.; Moellmann, J.; Grimme, S. Computation of accurate excitation energies for large organic molecules with double-hybrid density functionals. Phys. Chem. Chem. Phys. 2009, 11, 46114620 .

(29) Brémond, É.; Adamo, C. Seeking for parameter-free double-hybrid functionals: the PBE0-DH model. J. Chem. Phys. 2011, 135, 024106.

(30) Chai, J.-D.; Mao, S.-P. Seeking for reliable double-hybrid density functionals without fitting parameters: the PBE0-2 functional. Chem. Phys. Lett. 2012, 538, 121-125.

(31) Goerigk, L.; Grimme, S. Double-hybrid density functionals. Wiley Interdiscip. 
Rev.: Comput. Mol. Sci. 2014, 4, 576600.

(32) Najibi, A.; Casanova-Páez, M.; Goerigk, L. Analysis of recent BLYPand PBE-based range-separated doublehybrid density functional approximations for main-group thermochemistry, kinetics, and noncovalent interactions. $J$. Phys. Chem. A 2021, 125, 4026-4035.

(33) Goerigk, L.; Grimme, S. Calculation of electronic circular dichroism spectra with time-dependent double-hybrid density functional theory. J. Phys. Chem. A 2009, 113, 767-776.

(34) Goerigk, L.; Grimme, S. Assessment of TD-DFT methods and of various spin scaled CIS(D) and CC2 versions for the treatment of low-lying valence excitations of large organic dyes. J. Chem. Phys. 2010, 132, 184103.

(35) Goerigk, L.; Grimme, S. Double-hybrid density functionals provide a balanced description of excited ${ }^{1} \mathrm{~L}_{\mathrm{a}}$ and ${ }^{1} \mathrm{~L}_{\mathrm{b}}$ states in polycyclic aromatic hydrocarbons. $J$. Chem. Theory Comput. 2011, 7, 32723277 .

(36) Goerigk, L.; Kruse, H.; Grimme, S. Comprehensive chiroptical spectroscopy; Wiley-Blackwell, 2012; pp 643-673.

(37) Meo, F. D.; Trouillas, P.; Adamo, C.; Sancho-García, J. C. Application of recent double-hybrid density functionals to low-lying singlet-singlet excitation energies of large organic compounds. $J$. Chem. Phys. 2013, 139, 164104.

(38) Momeni, M. R.; Brown, A. A local CC2 and TDA-DFT double hybrid study on BODIPY/aza-BODIPY dimers as heavy atom free triplet photosensitizers for photodynamic therapy applications. J. Phys. Chem. A 2016, 120, 2550-2560.

(39) Schwabe, T.; Goerigk, L. Timedependent double-hybrid density functionals with spin-component and spin-opposite scaling. J. Chem. Theory Comput. 2017, 13, 4307-4323.

(40) Casanova-Páez, M.; Dardis, M. B.; Goerigk, L. $\omega$ B2PLYP and $\omega$ B2GPPLYP: the first two double-hybrid density functionals with long-range correction optimized for excitation energies. J. Chem. Theory Comput. 2019, 15, 4735-4744.

(41) Casanova-Páez, M.; Goerigk, L. Assessing the Tamm-Dancoff approximation, singlet-singlet, and singlet-triplet excitations with the latest long-range corrected double-hybrid density functionals. J. Chem. Phys. 2020, 153, 064106.

(42) Goerigk, L.; Casanova-Páez, M. The trip to the density functional theory zoo continues: making a case for time-dependent double hybrids for excited-state problems. Aust. J. Chem. 2021, 74, 3-15.

(43) Casanova-Páez, M.; Goerigk, L. Global double hybrids do not work for charge transfer: A comment on "double hybrids and time-dependent density functional theory: an implementation and benchmark on charge transfer excited states". J. Comput. Chem. 2021, 42, 528-533.

(44) Christiansen, O.; Koch, H.; Jørgensen, P. The second-order approximate coupled cluster singles and doubles model CC2. Chem. Phys. Lett. 1995, 243, 409-418.

(45) Hellweg, A.; Grün, S. A.; Hättig, C. Benchmarking the performance of spin-component scaled CC2 in ground and electronically excited states. Phys. Chem. Chem. Phys. 2008, 10, 4119.

(46) Goerigk, L.; Grimme, S. Quantum chemical investigation of exciton coupling: super-molecular calculations of a merocyanine dimer aggregate. ChemPhysChem 2008, 9, 2467-2470.

(47) Grimme, S. Improved second-order møller-plesset perturbation theory by separate scaling of parallel- and 
antiparallel-spin pair correlation energies. J. Chem. Phys. 2003, 118, 9095-9102.

(48) Jung, Y.; Lochan, R. C.; Dutoi, A. D.; Head-Gordon, M. Scaled opposite-spin second order Møller-Plesset correlation energy: an economical electronic structure method. J. Chem. Phys. 2004, 121, 9793-9802.

(49) Grimme, S.; Goerigk, L.; Fink, R. F. Spin-component-scaled electron correlation methods. Wiley Interdiscip. Rev.: Comput. Mol. Sci. 2012, 2, 886-906.

(50) Chai, J.-D.; Head-Gordon, M. Longrange corrected double-hybrid density functionals. J. Chem. Phys. 2009, 131, 174105 .

(51) Brémond, E.; Savarese, M.; SanchoGarcía, J. C.; Pérez-Jiménez, A. J.; Adamo, C. Quadratic integrand doublehybrid made spin-component-scaled. J. Chem. Phys. 2016, 144, 124104.

(52) Almlöf, J. Elimination of energy denominators in møller-plesset perturbation theory by a laplace transform approach. Chem. Phys. Lett. 1991, 181, 319-320.

(53) Tozer, D. J.; Amos, R. D.; Handy, N. C.; Roos, B. O.; Serrano-Andrés, L. Does density functional theory contribute to the understanding of excited states of unsaturated organic compounds? Mol. Phys. 1999, 97, 859-868.

(54) Tozer, D. J. Relationship between long-range charge-transfer excitation energy error and integer discontinuity in Kohn-Sham theory. J. Chem. Phys. 2003, 119, 12697-12699.

(55) Dreuw, A.; Head-Gordon, M. Failure of time-dependent density functional theory for long-range charge-transfer excited states:the zincbacteriochlorinbacteriochlorin and bacteriochlorophyllspheroidene complexes. J. Am. Chem. Soc. 2004, 126, 4007-4016.
(56) Becke, A. D. Density-functional exchange-energy approximation with correct asymptotic behavior. Phys. Rev. A 1988, 38, 3098-3100.

(57) Lee, C.; Yang, W.; Parr, R. G. Development of the colle-salvetti correlationenergy formula into a functional of the electron density. Phys. Rev. B 1988, 37, 785-789.

(58) Pishchalnikov, R. Y.; Yaroshevich, I. A.; Slastnikova, T. A.; Ashikhmin, A. A.; Stepanov, A. V.; Slutskaya, E. A.; Friedrich, T.; Sluchanko, N. N.; Maksimov, E. G. Structural peculiarities of keto-carotenoids in water-soluble proteins revealed by simulation of linear absorption. Phys. Chem. Chem. Phys. 2019, 21, 25707-25719.

(59) Pollice, R.; Friederich, P.; Lavigne, C.; dos Passos Gomes, G.; Aspuru-Guzik, A. Organic molecules with inverted gaps between first excited singlet and triplet states and appreciable fluorescence rates. Matter 2021, 4, 1654-1682.

(60) Suleiman, O.; Panthi, D.; Adeyiga, O.; Odoh, S. O. Methane C-H activation by $\left[\mathrm{Cu}_{2} \mathrm{O}\right]^{2+}$ and $\left[\mathrm{Cu}_{3} \mathrm{O}_{3}\right]^{2+}$ in copperexchanged zeolites: computational analysis of redox chemistry and X-ray absorption spectroscopy. Inorg. Chem. 2021, 60, 6218-6227.

(61) Gao, Z.; Chen, S.; Bai, Y.; Wang, M.; Liu, X.; Yang, W.; Li, W.; Ding, X.; Yao, J. A new perspective for evaluating the photoelectric performance of organic-inorganic hybrid perovskites based on the DFT calculations of excited states. Phys. Chem. Chem. Phys. 2021, 23, 11548-11556.

(62) Yaroshevich, I. A. et al. Role of hydrogen bond alternation and charge transfer states in photoactivation of the Orange Carotenoid Protein. Commun. Biol. 2021, 4, 539 . 
(63) Puget, M.; Shcherbakov, V.; Denisov, S.; Moreau, P.; Dognon, J.-P.; Mostafavi, M.; Le Caër, S. Reaction mechanisms of the degradation of $\mathrm{flu}-$ oroethylene carbonate, an additive of lithium-ion batteries, unraveled by radiation chemistry. Chem. Eur. J. 2021, $n / a, \mathrm{n} / \mathrm{a}$.

(64) Curtis, K.; Panthi, D.; Odoh, S. O. Time-dependent density functional theory study of copper(II) oxo active sites for methane-to-methanol conversion in zeolites. Inorg. Chem. 2021, 60, 1149 1159 .

(65) Brémond, É.; Sancho-García, J. C.; Pérez-Jiménez, Á. J.; Adamo, C. Communication: double-hybrid functionals from adiabatic-connection: the QIDH model. J. Chem. Phys. 2014, 141, 031101.

(66) Vintonyak, V. V.; Warburg, K.; Kruse, H.; Grimme, S.; Hübel, K.; Rauh, D.; Waldmann, H. Identification of thiazolidinones spiro-fused to indolin2-ones as potent and selective inhibitors of the mycobacterium tuberculosis protein tyrosine phosphatase B. Angew. Chem. Int. Ed. 2010, 49, 5902-5905.

(67) Send, R.; Valsson, O.; Filippi, C. Electronic excitations of simple cyanine dyes: reconciling density functional and wave function methods. J. Chem. Theory Comput. 2011, 7, 444-455.

(68) Markworth, P. B.; Adamson, B. D.; Coughlan, N. J. A.; Goerigk, L.; Bieske, E. J. Photoisomerization action spectroscopy: flicking the protonated merocyanine-spiropyran switch in the gas phase. Phys. Chem. Chem. Phys. 2015, 17, 25676-25688.

(69) Prlj, A.; Sandoval-Salinas, M. E.; Casanova, D.; Jacquemin, D.; Corminboeuf, C. Low-lying $\pi \pi^{*}$ states of heteroaromatic molecules: A challenge for excited state methods. J. Chem. Theory Comput. 2016, 12, 2652-2660.

(70) Alipour, M. On the performance of timedependent double-hybrid density functionals for description of absorption and emission spectra of heteroaromatic compounds. Theor Chem Acc 2016, 135, 18.

(71) Sancho-García, J. C.; Adamo, C.; PérezJiménez, A. J. Describing excited states of $[\mathrm{n}]$ cycloparaphenylenes by hybrid and double-hybrid density functionals: from isolated to weakly interacting molecules. Theo. Chem. Acc. 2016, 135, 25.

(72) Brémond, É.; Savarese, M.; PérezJiménez, Á. J.; Sancho-García, J. C.; Adamo, C. Speed-up of the excitedstate benchmarking: double-hybrid density functionals as test cases. J. Chem. Theory Comput. 2017, 13, 5539-5551.

(73) Ottochian, A.; Morgillo, C.; Ciofini, I.; Frisch, M. J.; Scalmani, G.; Adamo, C. Double hybrids and time-dependent density functional theory: an implementation and benchmark on charge transfer excited states. J. Comput. Chem. 2020, 41, 1242-1251.

(74) Hernández-Martínez, L.; Brémond, E.; Pérez-Jiménez, A. J.; San-Fabián, E.; Adamo, C.; Sancho-García, J. C. Nonempirical (double-hybrid) density functionals applied to atomic excitation energies: A systematic basis set investigation. Int. J. Quantum Chem. 2020, 120, e26193.

(75) Brémond, É.; Ottochian, A.; PérezJiménez, Á. J.; Ciofini, I.; Scalmani, G.; Frisch, M. J.; Sancho-García, J. C.; Adamo, C. Assessing challenging intraand inter-molecular charge-transfer excitations energies with double-hybrid density functionals. J. Comput. Chem. 2021, 42, 970-981.

(76) Sears, J. S.; Koerzdoerfer, T.; Zhang, C.R.; Brédas, J.-L. Communication: orbital instabilities and triplet states from 
time-dependent density functional theory and long-range corrected functionals. J. Chem. Phys. 2011, 135, 151103.

(77) Peach, M. J. G.; Tozer, D. J. Overcoming low orbital overlap and triplet instability problems in TDDFT. J. Phys. Chem. A 2012, 116, 9783-9789.

(78) Peach, M. J.; Warner, N.; David J. Tozer, On the triplet instability in TDDFT. Mol. Phys. 2013, 111, 1271-1274.

(79) Rangel, T.; Hamed, S. M.; Bruneval, F.; Neaton, J. B. An assessment of low-lying excitation energies and triplet instabilities of organic molecules with an ab initio bethe-salpeter equation approach and the Tamm-Dancoff approximation. J. Chem. Phys. 2017, 146, 194108.

(80) Mester, D.; Kállay, M. A simple rangeseparated double-hybrid density functional theory for excited states. J. Chem. Theory Comput. 2021, 17, 927-942.

(81) Marques, M.; Gross, E. Time-dependent density functional theory. Annu. Rev. Phys. Chem. 2004, 55, 427-455.

(82) Marques, M. A. L.; Ullrich, C. A.; Nogueira, F.; Rubio, A.; Burke, K.; Gross, E. K. U. Time-dependent density functional theory; Lecture notes in physics; Springer-Verlag, 2006.

(83) Mark E. Casida, In Recent advances in density functional methods; Chong, D. P., Ed.; World Scientific Singapore, 1995; pp 155-192.

(84) Gross, E. K. U.; Kohn, W. Timedependent density-functional theory. $A d$ van. Quantum Chem. 1990, 21, 255-291.

(85) McLachlan, A. D.; Ball, M. A. Timedependent Hartree-Fock theory for molecules. Rev. Mod. Phys. 1964, 36, 844-855.

(86) Iikura, H.; Tsuneda, T.; Yanai, T.; Hirao, K. A long-range correction scheme for generalized-gradient-approximation exchange functionals. J. Chem. Phys. 2001, 115, 3540-3544.

(87) Møller, C.; Plesset, M. S. Note on an approximation treatment for many-electron systems. Phys. Rev. 1934, 46, 618-622.

(88) Sharkas, K.; Toulouse, J.; Savin, A. Double-hybrid density-functional theory made rigorous. J. Chem. Phys. 2011, $134,064113$.

(89) Grimme, S.; Izgorodina, E. I. Calculation of 0-0 excitation energies of organic molecules by CIS(D) quantum chemical methods. Chem. Phys. 2004, 305, 223230 .

(90) Rhee, Y. M.; Head-Gordon, M. Scaled second-order perturbation corrections to configuration interaction singles: efficient and reliable excitation energy methods. J. Phys. Chem. A 2007, 111, 53145326 .

(91) Neese, F. The ORCA program system. Wiley Interdiscip. Rev.: Comput. Mol. Sci. 2012, 2, 73-78.

(92) Neese, F. Software update: the ORCA program system, version 4.0. Wiley Interdiscip. Rev.: Comput. Mol. Sci. 2018, 8, e1327.

(93) Valeev, E. F. Libint: A library for the evaluation of molecular integrals of many-body operators over gaussian functions. 2020,

(94) Vahtras, O.; Almlöf, J.; Feyereisen, M. Integral approximations for LCAO-SCF calculations. Chem. Phys. Lett. 1993, 213, $514-518$.

(95) Weigend, F.; Köhn, A.; Hättig, C. Efficient use of the correlation consistent basis sets in resolution of the identity MP2 calculations. J. Chem. Phys 2002, 116, 3175-3183. 
(96) Chai, J.-D.; Head-Gordon, M. Systematic optimization of long-range corrected hybrid density functionals. J. Chem. Phys. 2008, 128, 084106.

(97) Brémond, É.; Savarese, M.; PérezJiménez, Á. J.; Sancho-García, J. C.; Adamo, C. Range-separated doublehybrid functional from nonempirical constraints. J. Chem. Theory Comput. 2018, 14, 4052-4062.

(98) Leang, S. S.; Zahariev, F.; Gordon, M. S. Benchmarking the performance of timedependent density functional methods. $J$. Chem. Phys. 2012, 136, 104101.

(99) Loos, P.-F.; Galland, N.; Jacquemin, D. Theoretical $0-0$ energies with chemical accuracy. J. Phys. Chem. Lett. 2018, 9, 4646-4651.

(100) Grimme, S. In Reviews in computational chemistry; Lipkowitz, K. B., Boyd, D. B., Eds.; Wiley-VCH: New York, 2004; Vol. 20, pp. 153-218.

(101) Perdew, J. P. Density-functional approximation for the correlation energy of the inhomogeneous electron gas. Phys. Rev. B 1986, 33, 8822-8824.

(102) Vosko, S. H.; Wilk, L.; Nusair, M. Accurate spin-dependent electron liquid correlation energies for local spin density calculations: a critical analysis. Can. J. Phys. 1980, 58, 1200-1211.

(103) Koch, H.; Christiansen, O.; Jørgensen, P.; Sanchez de Merás, A. M.; Helgaker, T. The CC3 model: an iterative coupled cluster approach including connected triples. J. Chem. Phys 1997, 106, 1808-1818.

(104) Dunning, T. H. Gaussian basis sets for use in correlated molecular calculations. I. the atoms boron through neon and hydrogen. J. Chem. Phys. 1989, 90, 10071023 .
(105) Kendall, R. A.; Dunning, T. H.; Harrison, R. J. Electron affinities of the first-row atoms revisited. systematic basis sets and wave functions. J. Chem. Phys. 1992, 96, 6796-6806.

(106) Brémond, É.; Pérez-Jiménez, Á. J.; Sancho-García, J. C.; Adamo, C. Rangeseparated hybrid density functionals made simple. J. Chem. Phys. 2019, 150, 201102.

(107) Paci, I.; Johnson, J. C.; Chen, X.; Rana, G.; Popović, D.; David, D. E.; Nozik, A. J.; Ratner, M. A.; Michl, J. Singlet fission for dye-sensitized solar cells: can a suitable sensitizer be found? J. Am. Chem. Soc. 2006, 128, 1654616553.

(108) Wen, J.; Havlas, Z.; Michl, J. Captodatively stabilized biradicaloids as chromophores for singlet fission. J. Am. Chem. Soc. 2015, 137, 165-172.

(109) Grotjahn, R.; Maier, T. M.; Michl, J.; Kaupp, M. Development of a TDDFTbased protocol with local hybrid functionals for the screening of potential singlet fission chromophores. J. Chem. Theory Comput. 2017, 13, 4984-4996.

(110) Masoomi-Godarzi, S.; Liu, M.; Tachibana, Y.; Goerigk, L.; Ghiggino, K. P.; Smith, T. A.; Jones, D. J. Solution-processable, solid state donoracceptor materials for singlet fission. Adv. Energy Mater. 2018, 8, 1801720.

(111) Masoomi-Godarzi, S.; Liu, M.; Tachibana, Y.; Mitchell, V. D.; Goerigk, L.; Ghiggino, K. P.; Smith, T. A.; Jones, D. J. Liquid crystallinity as a self-assembly motif for high-efficiency, solution-processed, solid-state singlet fission materials. Adv. Energy Mater. 2019, 9, 1901069.

(112) Christiansen, O.; Koch, H.; Jørgensen, P. Perturbative triple excitation corrections 
to coupled cluster singles and doubles excitation energies. J. Chem. Phys. 1996, 105, 1451-1459.

(113) Loos, P.-F.; Scemama, A.; Blondel, A.; Garniron, Y.; Caffarel, M.; Jacquemin, D. A mountaineering strategy to excited states: highly accurate reference energies and benchmarks. $J$. Chem. Theory Comput. 2018, 14, 43604379.

(114) Loos, P.-F.; Scemama, A.; BoggioPasqua, M.; Jacquemin, D. Mountaineering strategy to excited states: highly accurate energies and benchmarks for exotic molecules and radicals. J. Chem. Theory Comput. 2020, 16, 3720-3736.

(115) Schäfer, A.; Horn, H.; Ahlrichs, R. Fully optimized contracted gaussian basis sets for atoms Li to Kr. J. Chem. Phys. 1992, 97, 2571-2577.

(116) Angeli, C.; Pastore, M. The lowest singlet states of octatetraene revisited. J. Chem. Phys. 2011, 134, 184302.

(117) Platt, J. R. Classification of spectra of cata-condensed hydrocarbons. J. Chem. Phys. 1949, 17, 484-495.

(118) Lopata, K.; Reslan, R.; Kowalska, M.; Neuhauser, D.; Govind, N.; Kowalski, K. Excited-state studies of polyacenes: A comparative picture using EOMCCSD, CR-EOMCCSD(T), rangeseparated (LR/RT)-TDDFT, TD-PM3, and TD-ZINDO. J. Chem. Theory Comput. 2011, 7, 3686-3693.

(119) Grimme, S.; Parac, M. Substantial errors from time-dependent density functional theory for the calculation of excited states of large $\pi$ systems. ChemPhysChem 2003, 4, 292-295.

(120) Parac, M.; Grimme, S. A TDDFT study of the lowest excitation energies of polycyclic aromatic hydrocarbons. Chem. Phys. 2003, 292, $11-21$.
(121) Marian, C. M.; Gilka, N. Performance of the density functional Theory/Multireference configuration interaction method on electronic excitation of extended $\pi$-systems. J. Chem. Theory Comput. 2008, 4, 1501-1515.

(122) Richard, R. M.; Herbert, J. M. Timedependent density-functional description of the 1La state in polycyclic aromatic hydrocarbons: charge-transfer character in disguise? J. Chem. Theory Comput. 2011, 7, 1296-1306.

(123) Kowalski, K.; Piecuch, P. New coupledcluster methods with singles, doubles, and noniterative triples for high accuracy calculations of excited electronic states. J. Chem. Phys. 2004, 120, 1715-1738.

(124) Jacquemin, D.; Duchemin, I.; Blase, X. Is the Bethe-Salpeter formalism accurate for excitation energies? Comparisons with TD-DFT, CASPT2, and EOMCCSD. J. Phys. Chem. Lett. 2017, 8, 1524-1529.

(125) Serrano-Andrés, L.; Fülscher, M. P. Theoretical study of the electronic spectroscopy of peptides. III. charge-transfer transitions in polypeptides. J. Am. Chem. Soc. 1998, 120, 10912-10920.

(126) Peach, M. J. G.; Benfield, P.; Helgaker, T.; Tozer, D. J. Excitation energies in density functional theory: an evaluation and a diagnostic test. J. Chem. Phys 2008, 128, 044118.

(127) Weigend, F.; Ahlrichs, R. Balanced basis sets of split valence, triple zeta valence and quadruple zeta valence quality for $\mathrm{h}$ to rn: design and assessment of accuracy. Phys. Chem. Chem. Phys. 2005, 7, 3297-3305. 
Graphical TOC Entry

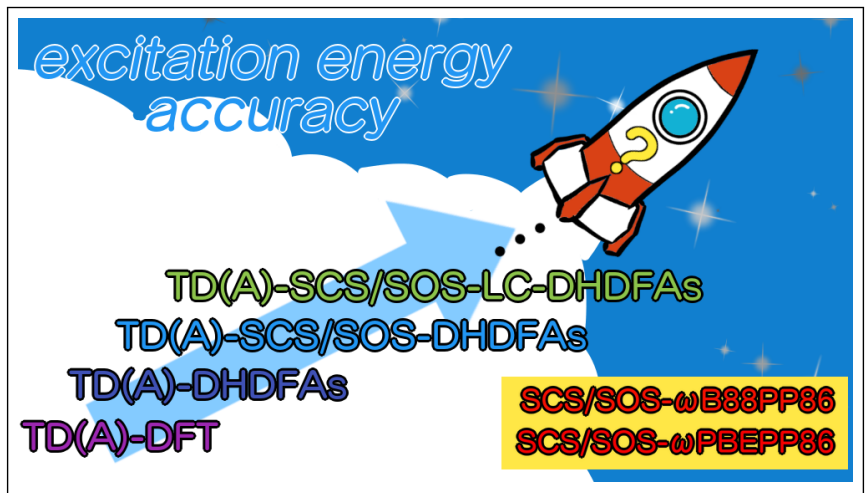

